\title{
تصميم نموذج دعم قرار الاستبدال باستعمال المحاكاة
}

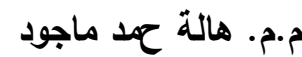

كلية الادارة والاقتصاد

جامعة بغدد

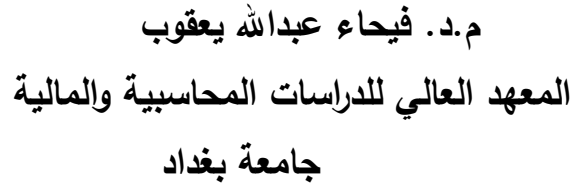

أ.د.صباح مجيد النجار

كلية الادارة والاقتصاد

جامعة بغداد

\section{Abstract}

Analysis of decisions regarding the replacement of old production

equipments and technologies with new ones includes defining the required productive capacity levels and provision of the necessary financial needs to purchase them. Researchers have submitted a set of methods that deal with the problems of replacement, which were based on a set of assumptions. They sometimes hypothesized the consistency of the productive age of equipments, and elsewhere they were limited to the treatment of single equipment. Such assumptions make these methods irrelevant for making the replacement decisions especially those equipments whose age is a random variable such transport buses. Thus the researchers attempted to build a quantitative method characterized by flexibility to simulate the operation of bus replacement in the future. The results of the simulation were used to test the correlation between the number of replacements and the planned age of the productive equipments (the buses). The test revealed that there is no significant relationship between the number of replacements and the planned age of the equipments, in other words the destiny of the transport bus is independent of its planned age, and that the need for replacement is not characterized by a linear relationship with the planned age of the equipment. The research recommends the adoption of the Replacement Decision Support Tool developed in this study for analyzing the replacement decisions due to the nature of the outputs provided by the model which supply the user with the basic themes for analyzing the replacement decisions.

\section{المقدمـة}

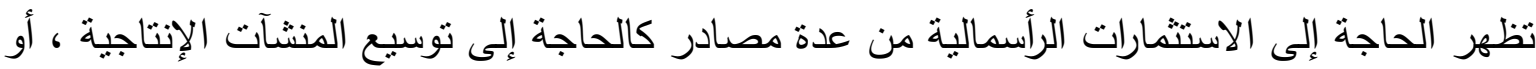

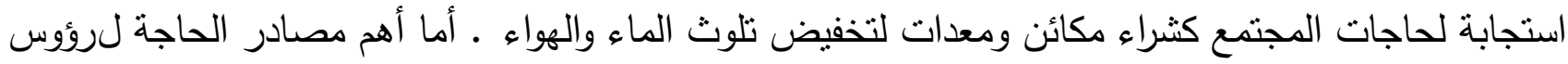

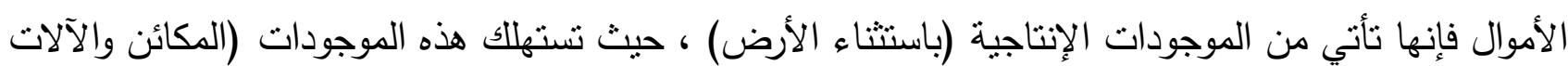

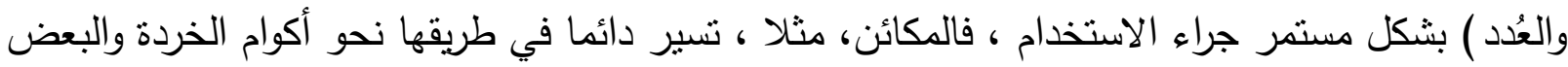

$$
\begin{aligned}
& \text { الآخر منها يصل إلى نهاية عمره الإنتاجي في نهاية لك ل سنة ، وأحيانا تصل الموجودات الإنتاجية إلى نهاية }
\end{aligned}
$$

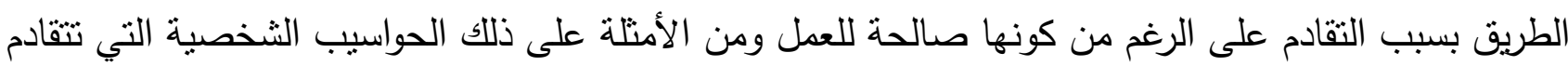

$$
\begin{aligned}
& \text { بسرعة. }
\end{aligned}
$$


إن أولى الأسبقيات التي تتطلب الحجم الأكبر من الاستثمارات الرأسمالية هي الحاجة لاستمرار عمل

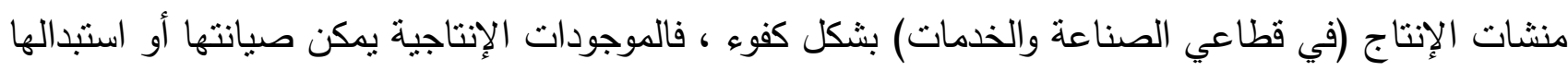
وبقدر تعلق الحاجة إلى رؤوس الأموال فان استبدال الموجودات يتطلب الجزء الأكبر من رؤوس الأموال المتاحة للثركة ، ولا تستهلك الموجودات الإنتاجية بأكملها مرة واحدة لان هذه الحالة لو تحققت فيكون من السهل معرفة الته تاريخ استبدالها ولكن بالمقابل تتضمن العديد من قرارات إدارة العمليات استثمار رؤوس أموال كبيرة ومن الأمثلة

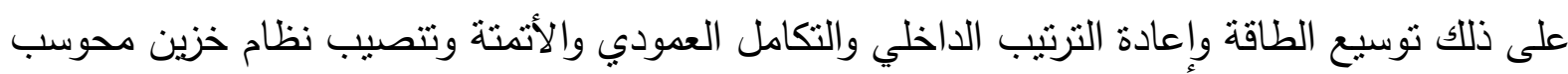
Cross-

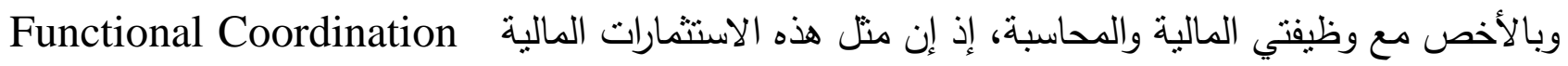

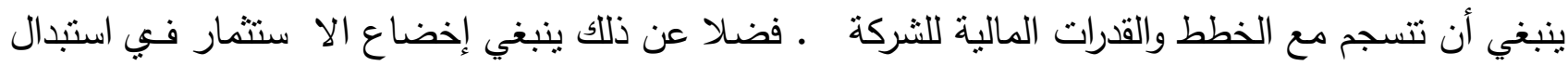

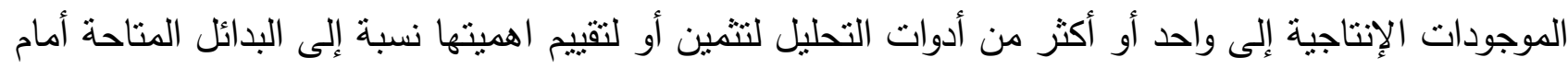

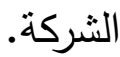

وفي الواقع قام الباحثون في مجال بحوث العمليات وإدارة العمليات مجموعة من الأساليب والنماذج

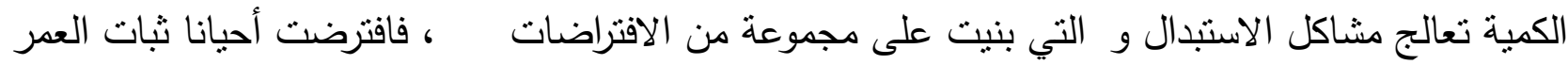

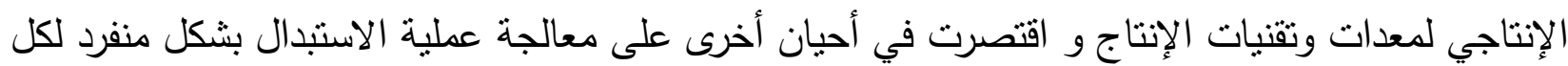

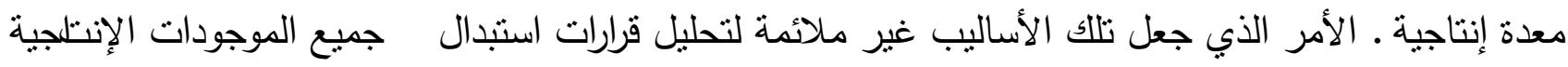

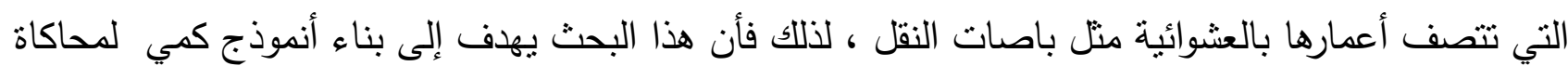

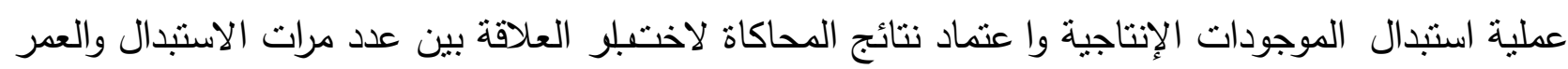

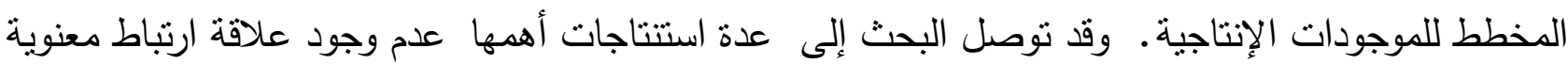
بين عدد مرات الاستبدال المحتملة والعمر المخطط للموجودات الإنتاجية ، وان الحاجة إلى الاستبدال لا تتسم بعلاقة خطية بالعمر المخطط للموجودات الإنتاجية ، لذلك يشجع الباحثون على استخدام نموذج دعم قرار الاستبدال في اتخاذ قرار استبدال الموجودات الإنتاجية.

\section{المبحث الأول}

استبدال الموجودات الإنتاجية: إطار نظري

\section{1- 1 - 1 تعريف الاستبدال وأهميته}

إذ يقصد بالإحلال Replacement ومصطلح الاستبدال Substitution لابد من التمييز بين مصطلح الإحلال التدريجي أي تصبح غير صالحة الدانتهاء العمر الفني لوسائل ومعدات الإنتاج نتيجة لاندثارها

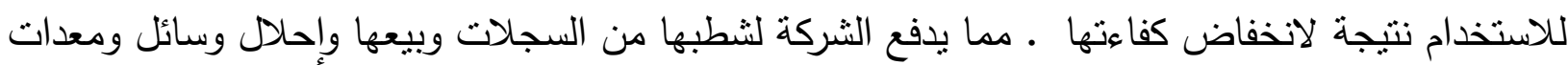

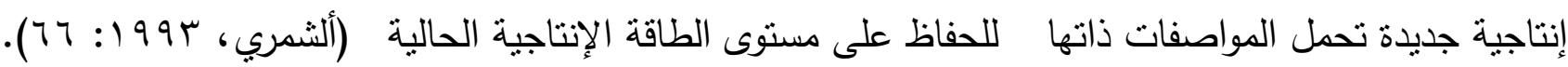

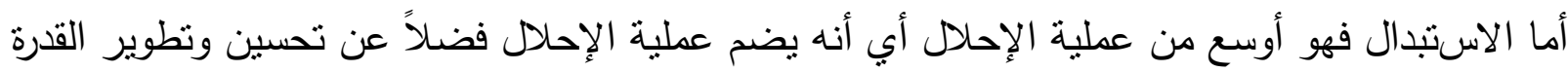
الإنتاجية وذلك لإدخاله معدات وتقنيات إنتاجية حديثة وذات مستوى تكنولوجي منطور ، فالاستبدال يأخذ بنظر الإني 
الاعتبار الظروف الاقتصادية المستقلية ومستوى التطور التكنولوجي المستمر، ويعتمد قرار الاستبدال على

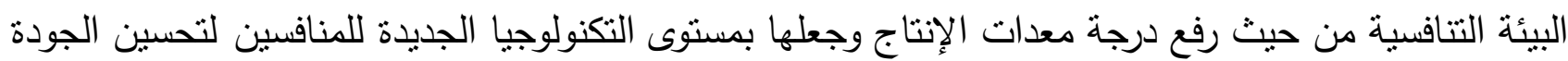
وزيادة المرونة وخفض التكاليف عن طريق خفض معدلات الصيانة والتلف والمع يب مما يدعم المكانة التنافسية • ولهذا فإن الاستبدال يكون ضمن مجالين هما (الحبيطي، (Russell and Taylor III, 1995: 295)للشركة $:(r+7:) 99 \mathrm{~V}$

أ- استبدال التقنيات الإنتاجية المشابهة في الغرض والاستخدام (عملية إحلال).

ب- استبدال التقنيات الإنتاجية غير المتثابهة في الغرض والاستخدام.

ونتيجة لما تقام يمكن تعريف الاستبدال بأنه قرار لتحديد أفضلية استبدال معدات وتقنيات الإنتاج الحالية مقابل أخرى، سواء كان لها ذات المواصفات والطاقة أو تختلف عنها وتأتي من عدة أسباب تؤثثر على ربحية وإنتاجية الثركة.

1- أسباب استبدال تقتيات الإنتاج

هي:-Bowman, 1999: 346) ل للاستبدال عدة أسباب تدفع الثركات للقيام به Deterioration and Depreciation

تتعرض معدات وتقنيات الإنتاج للتلف والاندثار نتيجة الاستخدام المستمر لها مما يجعل كلفة صيانتها

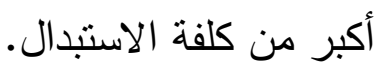

\section{Reduced Performance}

قد تكون كفاءة الآلات والمكائن غير كافية لمواجهة منطلبات الزبائن، أي انخفاض درجة الاعتماد عليها وانحراف مستوى جودة الإنتاج وانخفاض الإنتاجية.

\section{Abrupt Failure}

يتضمن الانهيار الرئيسي أو الأساسي الناتج عن التدمير نتيجة الحريق أو الفيضان أو الهزات الأرضية

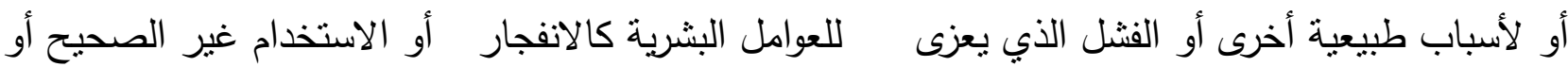

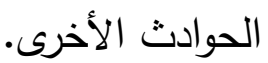

$$
\text { Costs }
$$

عند المقارنة بين كلف معدات الإنتاج القديمة ومعدات الإنتاج الجديدة قد يظهر بلبن المعدات القديمة تملك كلف عالية بسبب ارتفاع كلف التشغيل والصيانة (نتيجة للعطلات المستمرة)، وقد تظهر كلف أخرى نتيجة لانخفاض الكفاءة وتقدم العمر. 
تستخدم المعدات الإنتاجية لإنتاج السلع والخدمات لإشباع حاجات الأفراد ولكن عندما ينخفض الطلب على تلك السلع أو الخدمات أو يتغير تصميمها، تصبح المعدات القديمة غير اقتصادية لمواكبة المتطلبات الجديدة (Sullivan et al., 2000: 389).

\section{Obsolescence Technological}

المقصود بتقادم التكنولوجيا (هو الانخفاض في قيمة الموجود الفعلي عن قيمته الافترية بسبب التطورات التكنولوجية التي تغير القيمة الاقتصادية للموجودات (الثمري، ب99 1: 0 V). وهو حالة الاحتفاظ بالآلات والمعدات الإنتاجية القديمة في وقت يفضل فيه استبدالها إلا أن الوضع المالي للشركة لا يسمح بذلك. ، فالاندثار يظهر نتيجة Obsolescence والتقادم Depreciation وعليه يمكن التمييز بين الاندثار الاستخدام المستمر للآلة أو الماكنة، أما التقادم فيظهر نتيجة التطورات المستمرة التي تحصل في الجانب التقني والتكنولوجي والذي يهدف عادة لرفع وتحسين الطاقة الإنتاجية لمعدات وتقنيات الإنتاج ، وعلى هذا الأساس فإن :الانحدار في مستوى المعدات قد يكون ناتجاً من عدة عوامل Shamblin and Stevens, 1974: 85) -: التقادم الفني ، وارتفاع كلف الصيانة ، ارتفاع كلف النتخيل ، ودقة الإنتاج.

\section{- jRejection}

إن زيادة نسب المعيب ومخلفات الإنتاج في العملية الإنتاجية يؤدي إلى القيام بعملية استبدال تلك المعدات

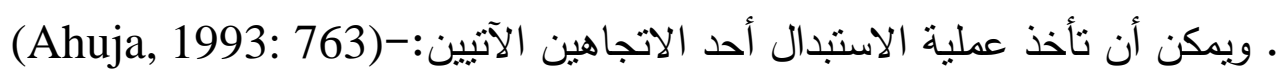

1- استبدال المكائن والمعدات التي تقل كفاءتها تدربجياً بمرور الزمن نتيجة الاستخدام المستمر •

ץ- استبدال الوحدات التي نتلف كلياً وبصورة مفاجئة كالمحركات والمصابيح بكافة أنواعها والبطاريات ، وتقسم (Shafer and Meredith, 1998: 790)-:

\section{- سياسة الاستبدال الفردبية}

إذ تستبدل الأجزاء عند وقوع العطل ولكل جزء عاطل بشكل فردي وذلك لارتفاع كلفة استبدال ذلك الجزء العاطل. - سباسة الاستبدال الجماعية

وهي السياسة التي يتم فيها استبدال جميع الأجزاء بعد فترة محددة من الوقت مثنلاً (• أثنهر ) سواء كان هناك عطل أم لا، إذ أن كلفة الاستبدال أقل من كلفة التصليح

(Reid and Stark, 1986: 23-27).

وتظهر العلاقة بين قرار الاستبدال وعملية تخطيط الطاقة الإنتاجية لتقنيات ومعدات الإنتاج من خلال التأثنر المباشر الذي يخلقه قرار استبدال المعدات الحالية (الطاقة الحالية ) على مستوى الطاقة المستقبلية المطلوبة لمواجهة الطلب. أن زيادة مستوى الطاقة الحالية والمتمثل بشراء مكائن ومعدات إنتاجية جديدة أو ذات طاقة إنتاجية عالية دون أن تقابلها حاجة فعلية سوف يحّرض الثركة لخسارة ناتجة عن تحليل غير دقيق لمستوى الطاقة ومن ثم استثمارات غير اقتصادية ـ أو انخفاض مستوى الطاقة نتيجة عملية الاستبدال دون أن تقابلها طاقة جديدة تستوعب الطلب الفعلي يؤدي إلى خسارة ناتجة من فقدان حصة سوقية . وتعدّ تحليلات التعادل من 
النماذج الرياضية المهمة في إدارة العمليات التي يعتمد عليها في اتخاذ القرارات المناسبة لإيجاد التوازن بين الطاقة والطلب (Slack et al., 2004: 374).

\section{ا - The Optimum Time of Replacement}

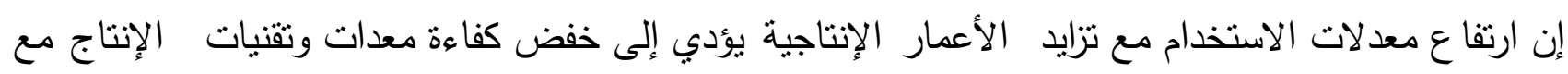

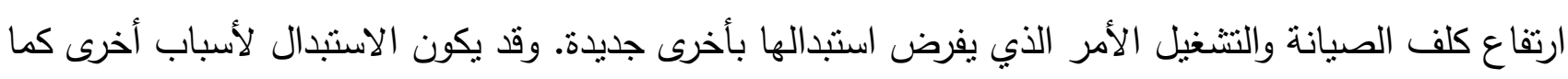

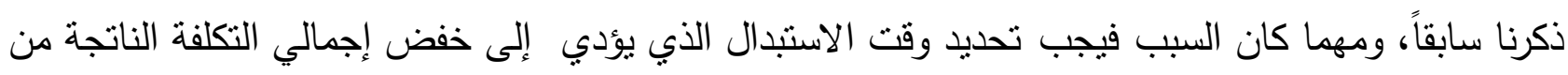

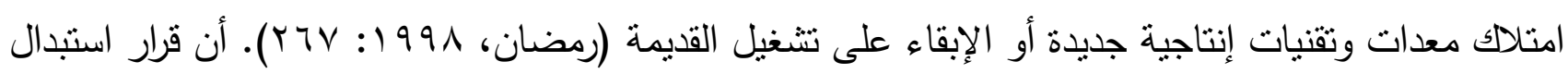

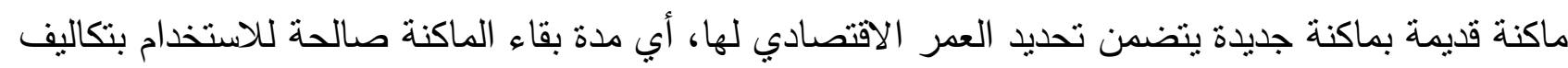

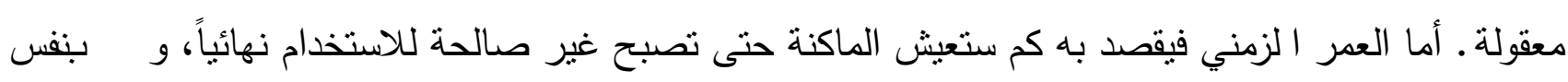

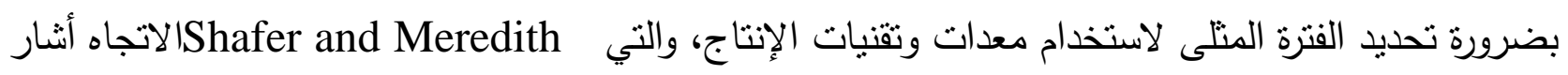

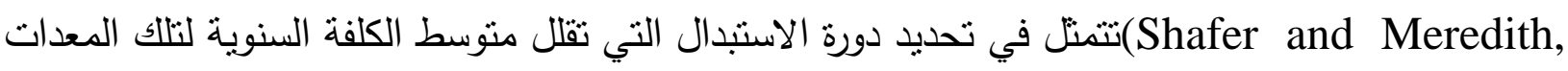

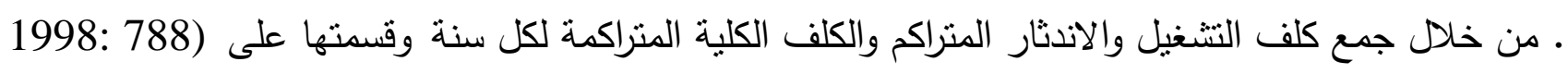
عدد سنوات العمر الإنتاجي والناتج المتحقق يمثل منوسط الكلف السنوية، حيث يتم تحديد سنة الاستبدال التي يكون عندها متوسط الكلفة السنوية أقل ما يكون ، إذ أن التكاليف ستزداد في السنة اللاحقة، ولذلك يتم تحديد

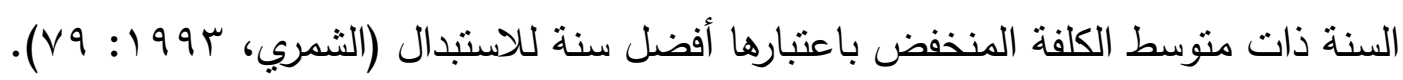

إن هذا الأسلوب يفترض أن حياة معدات وتقنيات الإنتاج ذات أعمار إنتاجية ثابتة، أو بمكن تقديرها

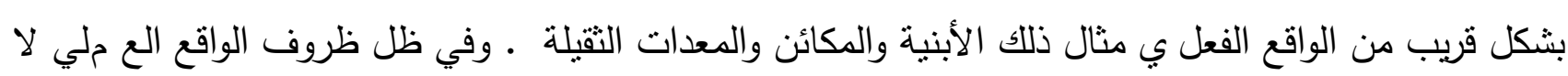

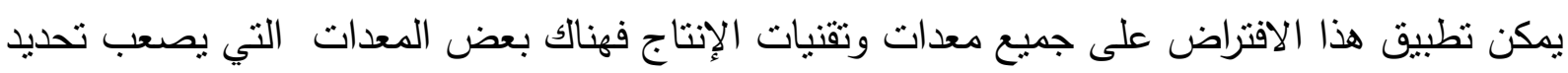

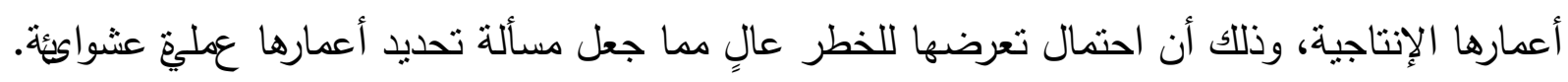

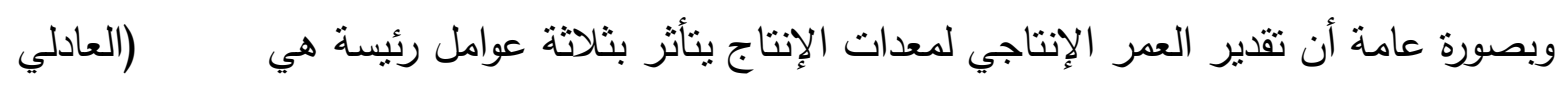
وآخرون،

Physical Depreciation or Deterioration

$$
\begin{gathered}
\text { rInadequacy } \\
\text { rObsolescence }
\end{gathered}
$$

وهناك مدلولات مختلفة لحياة معدات وتقنيات الإنتاج وهي (Bowman, 1999: 353).

\section{Physical Life}

هي المدة التي تبدأ من بداية عمل الآ لـة أو الماكنة لغاية عطلها أو تلفها، وهي عادة أطول قياساً

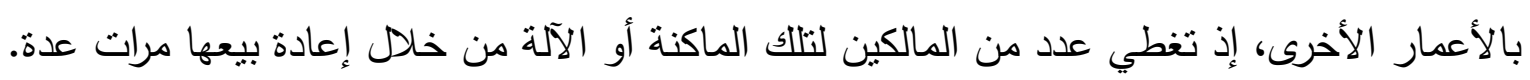
ثانيا- الحياة المحاسبية 
وتمثل الحياة الضريبية ويقصد بها الزمن الذي تُستَهلك فيها تقنيات الإنتاج وقد تعكس أو لا تعكس الحياة المادية.

\section{Oثالثا- الحياة الخاصة (المملوكة)}

هي المدة التي تمتلك خلالها الثركة تقنيات الإنتاج إذ يمكن شراء تقنيات إنتاجية مستخدمة أو مباعة قبل نهاية حياتها المادية.

\section{Economic Life}

وهي الحياة المتبقية والتي تصبح فيها كلفة تقنيات الإنتاج الحالية أكبر من كلفة البدائل، أي أن الحياة الإقتصادية هي أقل كلفة سنوية مقابل إنهاء حياة تقنيات الإنتاج الحالية واستبدالها بأخرى ذات كلف سنوية أ قل.

وعلى الرغم من ذلك تبقى مسألة تحديد وقت الاستبدال من الم شناكل المعقدة. فهي تعتمد على طبيعة معدات الإنتاج المستخدمة من ناحية، وعلى درجة توفر البيانات والمعلومات الخاصة بهذه المعدات من ناحية أخرى وعلى طريقة استخدام تلك التقنيات. وهناك جملة من البيانات التي ينبغي على الثركة دراستها عند إتخاذها لقرار الاستبدال والتي على ضوئها يمكن تحديد إذا كان من الأفضل القيام بالاستبدال أم لا.

أ- بيانات عن الآلات والمكائن القديمة

وتشمل هذه البيانات ما حققتهُ الآلة القديمة من تدفقات نقدية ومستويات تشغيل إضافة إلى تكاليف الصيانة وعدد السنوات التي مرت من عمرها الإنتاجي والمتبقي منه ، وكم ستحقق من تدفقات نقدية في المدة المتبقية من حياتها الإنتاجية وقيمتها كأنقاض في نهاية عمرها الإنتاجي.

ب- بيانات عن الآلات والمكائن الجديدة

وهي المعدات الإنتاجية التي لا تمنلكها الثركة لذا يجب أن تتوفر عنها بيانات من حيث إمكانيتها في استيعاب تكنولوجيا التقنيات الجديدة والمتطورة ومن حيث النوع والكفاءة ومتطلبات التشغيل إضافة إلى بيانات

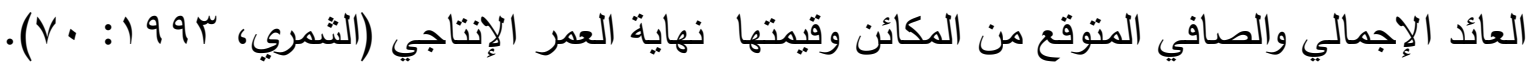
تخضع معدات وتقنيات الإنتاج لثلاثة جوانب رئيسة يتعلق الجانب الأول بتحديد كل فتها الاستثمارية،

والجانب الثاني يهتم بتخصيص هذه الكلفة على العمر الإنتاجي لتلك المعدات والذي يعرف محاسبياً بالإندثار ، أما الجانب الثالث فيرتبط بكلفة استبدال معدات وتقنيات الإنتاج وكيفية استبعادها وإزالتها من الخدمة. وينبغي على المنظمات أن تهتم بكيفي ة معالجة وتحليل كلف الاستبدال، وذللك لأهميتها والدور الذي تحتله في توفير المعلومات الضرورية التي تستقيد الإدارة منها في تحليل قراراتها الإنتاجية والاستثماربة فضلاً عن المستقبدين الآخرين.

وتعرف كلف الاستبدال بأنها " المصروفات المطلوية لاستبدال معدات إنتاجية قيد الاستعمال بمعدات "أخرى مماثلة أو مختلفة واعتبارا من موعد محدد (Gress, 1972: 3$)$. 
(Bowman, 1999: 364).

$$
\begin{gathered}
\text { I - الإخراج من الخدمة دون مقابل - Discarded } \\
\text { - البيع بمقابل نقدي Sold For Cash }
\end{gathered}
$$

ז Exchange For Another Equipment

Keeping Old Equipment as Backup

$$
\text { تحليل قرارات الاستبدال الثاني }
$$

r- 1 - تعيل قرارات الاستبدال باستخدام معايير الاستثمار المالي

\section{Replacement Decisions Analysis via Investment Criteria}

، أن قرارات الاستثمار تخصص وتتفق الأموال في الوقت الحاضر للحصول على عوائد في المستقبل

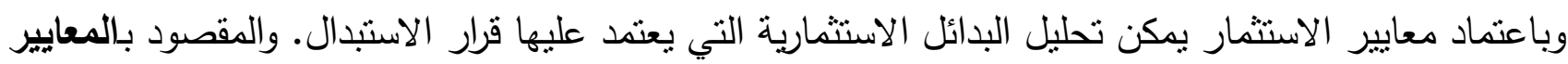

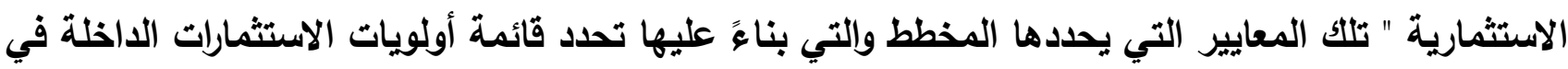

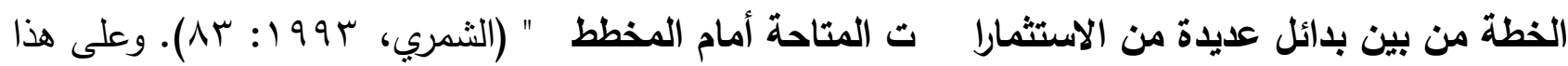

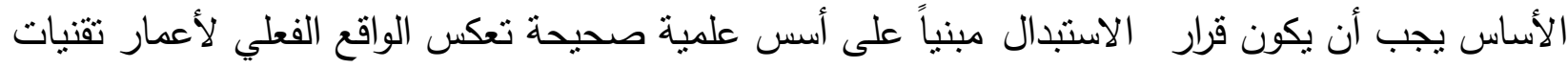
الإنتاج ومقدار العوائد المتحققة ضمن مدة حياتها، ومن خلال معايير الاستثمار يمكن إخضاع قرار الاستبدال

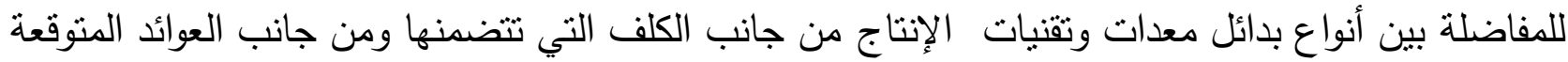
ولكل بديل في ضوء أعمارها الإنتاجية، مما يفرض على الثركة تحديد مستوى الطاقة المطلوب من حيث العدد

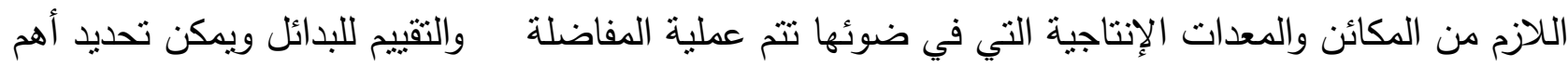
معايير الاستثمار بالآتي:-

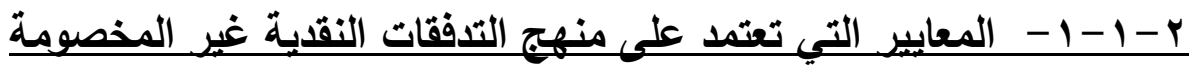

\section{Discounted Cash Flows (DCF)}

\section{Payback Period (PBP)}

من أكثر معايير التحليل إستخداماً وأسهاها حساباً، إذ يعتمد على العمر PBP يعُ معيار فترة الاسترداد

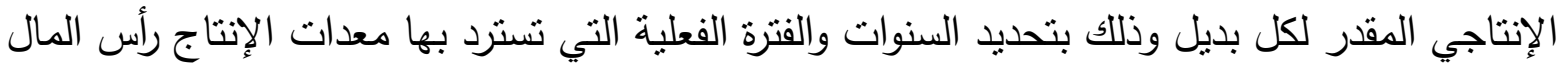
المستثر فيها ـ وتعرّف فترة الاسترداد بـ " عدد السنوات المتوقعة والمطلوية لإعادة ت غطية كلفة الاستثمار (Pandey, 1997: 336) .

• ويمكن حساب فنرة الاسترداد وفقاً للقاعدة الآتية:-

$$
P B P=\frac{I C}{A R} \text {. }
$$


IC = كلفة الاستثمار (Investment Cost).

AR = العائد السنوي (Annual Revenue) (Khan and Jain, 1982: 232)

وعلى الرغم من بساطة وشيوع استخدام هذا المعيار إلا أنه لا يخلو من بعض العيوب، أهمها تجاهل

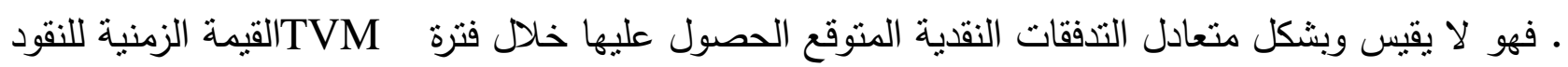

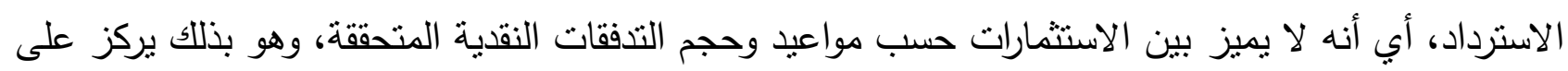

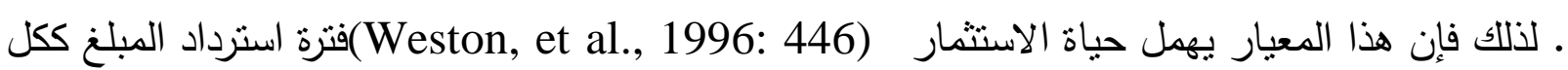

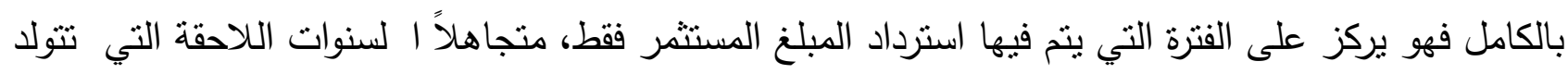

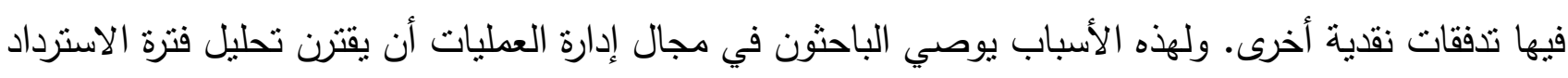

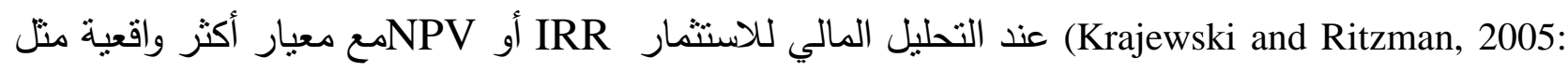
849).

في تحليل وتقييم البدائل مفيداً للاستثمار في المعدات والمكائن الإنتاجية، إذ إن PBP إن إنتخدام معيار

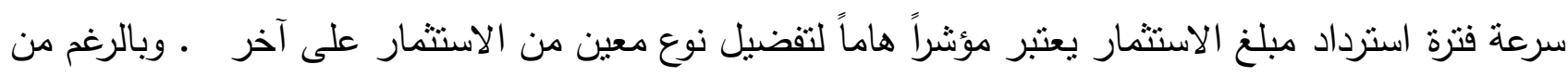

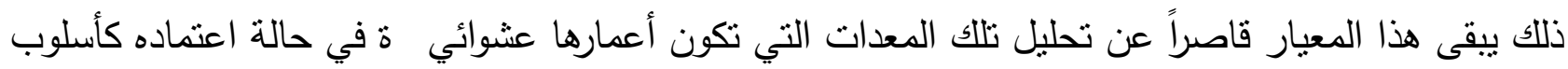
منفرد في عملية التحليل بدون دعمه بأسلوب آخر أكثر قابلية ومرونة.

\section{ثُانياً - معكوس فترة الاسترداد}

لتقدير معدل العائد لمعدات وتقنيات الإنتاج وبدون إتباع أسلوب خصم PBR يمكن استخدام معيار

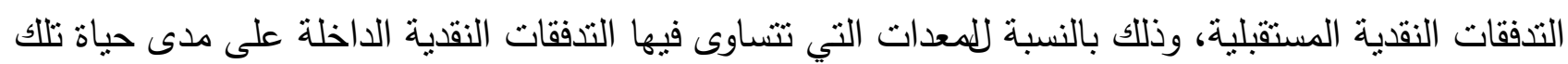

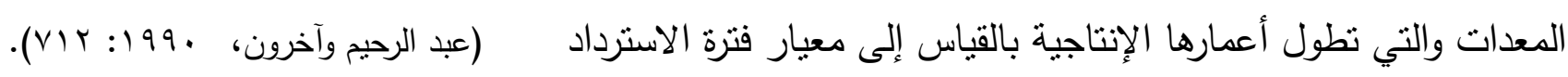
ويحسب وفق القاعدة الآتية:-

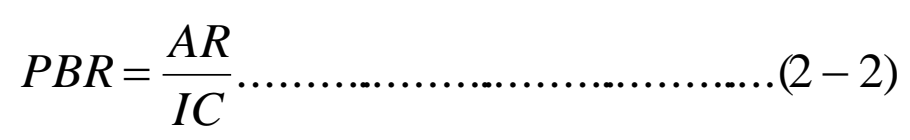

يوفر هذا المعيد كثيراً من الوقت والجهد في تقييم وتحليل بدائل الاستثمار ، أما أبرز عيوب هذا المعيار تتمنل باستخدامه التدفقات النقدية المتساوية والمنتظمة ولا يمكن تطبيقه في ظل التذفقات النقدية غير المنساوية

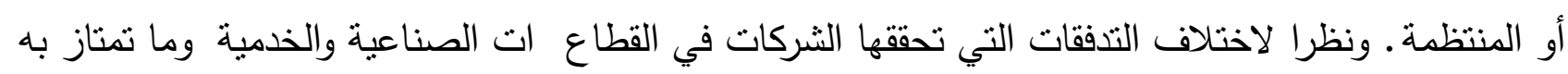

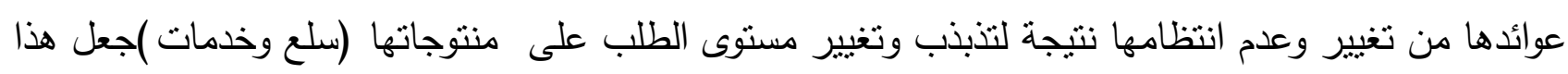
المعيار غير مناسب للتقييم والتحليل.

Accounting Rate Of Return 


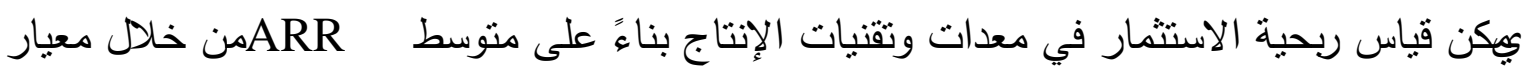

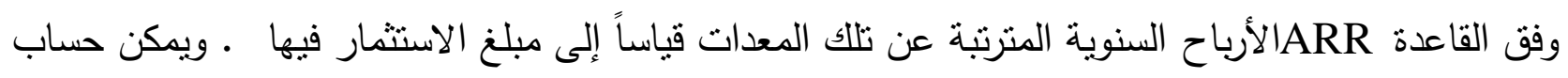

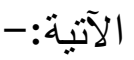

$$
A R R=\frac{A A P}{I C} .
$$

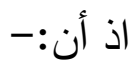

AAP = متوسط الربح المحاسبي (Average Accounting Profit).

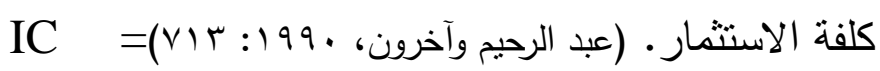

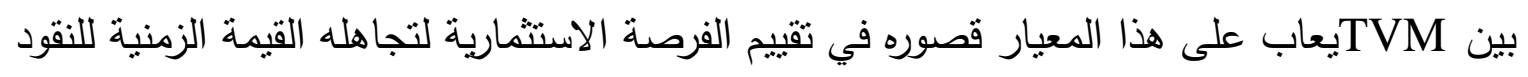
الفترات المختلفة.

ومن الجدير بالذكر أن المعايير الثلاثة السابقة تفترض أن عمر ا لموجود معلوم (أو متمثل بإيراداته

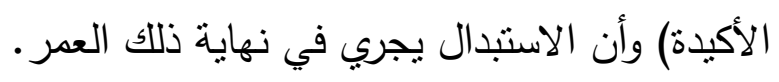

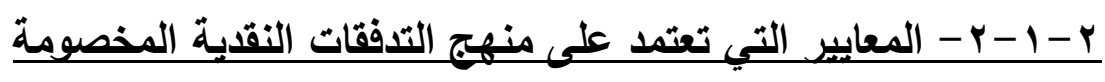

\section{Discounted Cash Flows (DCF)}

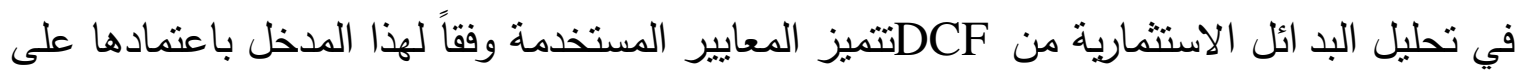

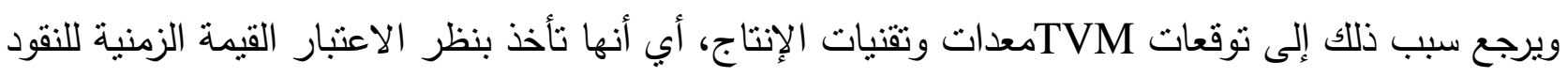

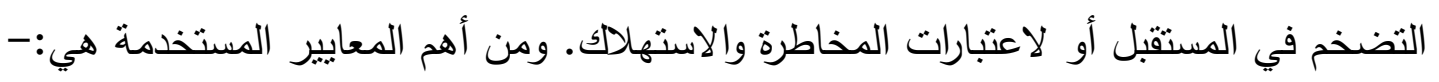

Net Present value

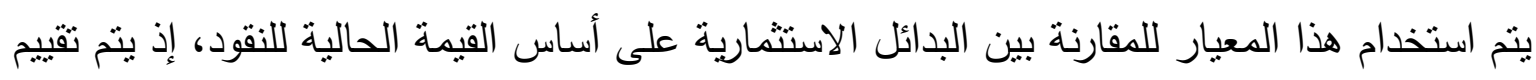

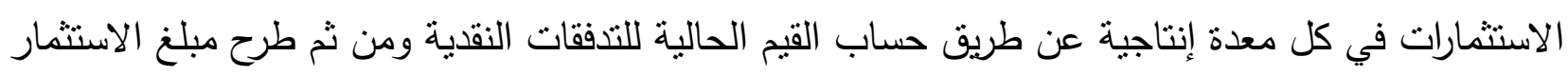
من مجموع القيم الحالية، ويمتل الفرق صافي القيمة الحالية (Krajewski and Ritzman,2005: 833).

ويحسب صافي القيمة الحالية وفق القاعدة الآتية:-

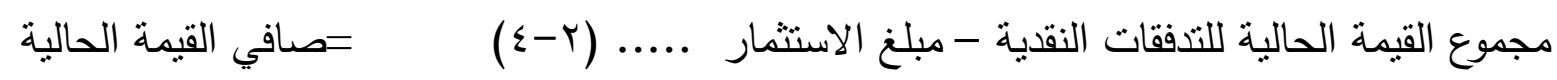

وبناءً على الننائج الدتحققة فإن القرار يخضع للمقارنة الآتية :-

صفر > NPV - n - n إذا كانت

يتم قبول البديل الاستثاري إذ أن مقدار التذفقات النقدية أكثر من الاحتياجات اللازمة لتنطية كلفة

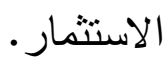

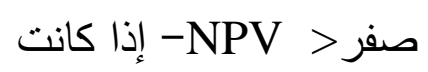

يتم رفض البيل وذلك لعدم جدوى الاستثمار فيه.

صفر = NPV - n إذا كانت 
للشركة الحرية بالقبول أو الرفض أي أ ن التذفقات النقدية مساوية لكلفة الاستثمار من دون تحقيق أية عوائد إضافية (Weston et al., 1996: 498).

ويحمل هذا المعيار مجموعة من المزايا أهمها:-

بنظر الاعتبار ITVM - يأخذ هذا المعيار القيمة الزمنية للنقود

r- يعدُ طريقة صحيحة للتحليل والتقييم لأنه يأخذ بنظر الاعتبار مجموع العوائد الناشئة عن الماكنة أو الآلة الأل الإنتاجية عبر سنوات حياتها (Pandey, 1997: 329).

عن طريق تحوير المقياس لأن النسبة تتغير مع تغير NPV- أن نسبة الخصم المتغيرة بمكن بناءها ضمن (Khan and Jain, 1982: 241) .

واحداً من أفضل معايير تقييم بدائل الاستثمار في إدارة العمليات NPV وعلى هذا الأساس يعـ معيار

(Heizer and Render, 2001: 262).

وعلى الرغم من هذه المزايا إلا أن ذلك لا يعني أنه يخلو من العيوب والتي من أهمها:-

INPV يتطلب بيانات أكثر من معيار PBP (Khan and Jain, 1982: 242).

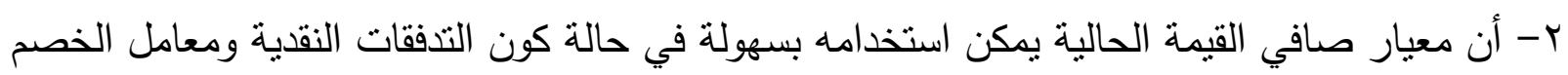

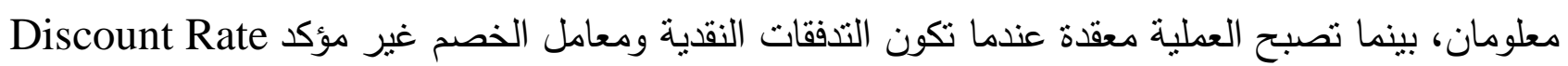
لا سيما عندما تكون أعمار معدات وتقنيات الإنتاج غير منساوية مما يجعل العملية معقدة.

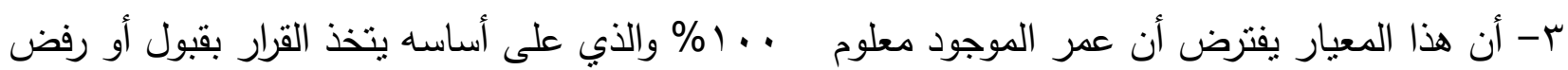
صفر > Pandey, 1997: 329).

\section{Internal Rate of Return}

هذا المعيار لا يختلف عن المعيار السابق فهو يأخذ القيمة الحالية للتدفقات النقدية في الاعتبار، وتتمنل

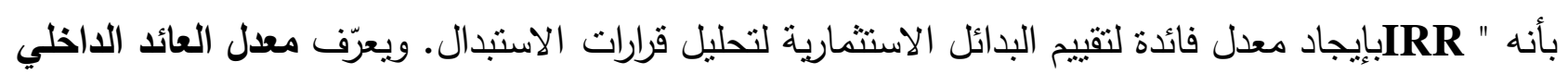
المعدل المتوقع أن تحققه الثركة على الاستثمار، فهو معدل الخصم المعادل للقيمة الحالية للتدفقات النقدية التانية

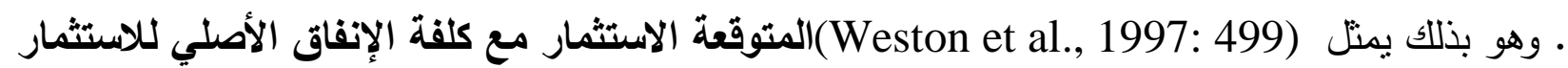
معدل الخصم الذي يكون عنده صافي القيمة الحالية للبديل الاستثماري الماكنة أو الآلة الإنتاجية ) مساوياً

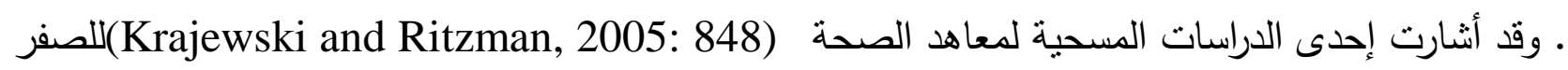
الوطنية في أميركا إلى إن هذا الأسلوب يلاقي رواجا كبيرا من قبل الثركات في اتخاذ قرارات الاستبدال (National Institutes of Health,1990,67).

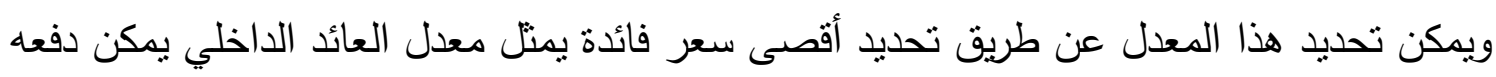
للأموال المستثمرة في استبدال الآلات والمعدات الإنتاجية ضمن فئل الفترة الزمنية المستخدمة فيها. وفق القواعد الآتية:-IRR وينم حساب التئه 
- في حالة كون التدفقات النقدية منتظمة ومتساوية خلال العمر الإنتاجي للماكنة أو الآلة يتم استخراج معامل

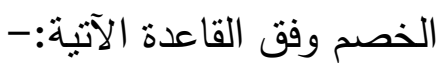

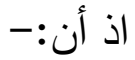

$$
\text { . (r-0) } \frac{\mathrm{IC}}{\mathrm{AR}} \mathrm{DF}=
$$

DF = معامل الخصم (Discount Factor)

(Bhattacharyya and Dearden, 1996: 659)

وبتطبيق القاعدة رقم (ץ-0) يتم الرجوع إلى جداول القيمة الحالية ذات الدفعات المنساوية والبحث عن معامل الخصم المستخرج، وبالنظر إلى العمر الإنتاجي المحدد يستمر البحث حتى يتم إيجاد سعر الفائدة المناسب وفقاً لمعامل الخصم.

بسبب - في حالة كون التدفقات النقدية غير منتظمة خلال العمر الإنتاجي، وهذه الحالة هي الأكثر شيوعاً IRR تذبذب وتغير الطلب مما يجعل العوائد غير منتظمة، فيتم إيجاد • فيتم تجربة عدة قيم لمعامل الخصم إلى أن يصبح Error (Krajewski and Ritzman, 2005:849) صافي القيمة الحالية للتدفقات النقدية مساوية لمبلغ الاستثمار .

يمتاز هذا المعيار بأنه يأخذ بنظر الاعتبار القيمة الزمنية للنقود ويهتم بالتدفقات النقدية الداخلة والخارجة

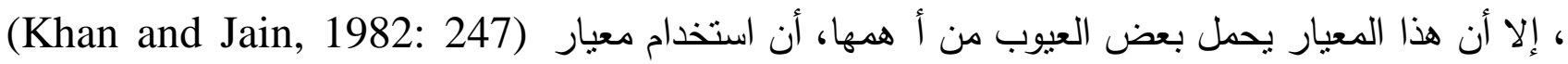

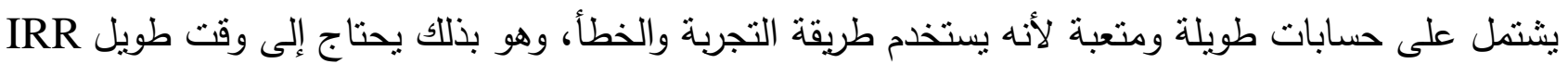
يقدم معدلات متعددة مما يؤدي إلى الى لحتى يتم الحصول على معدل الخصم المطلوب، كما أن معيار

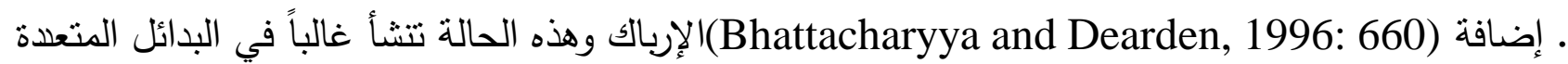
إلى افتراض ثبات العمر الإنتاجي لمعدات وتقنيات الإنتاج.

\section{Profitability Index (PI)}

وهو أحد المعايير المستخدمة في تحليل الاستثمارات في معدات وتقنيات الإنتاج ويحسب وفق القاعدة الآتية

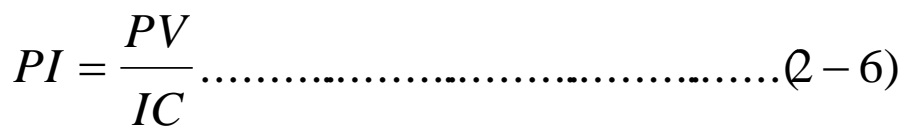

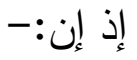

PV =القيمة الحالية للتدفقات النقدية.

أما حالة القبول والرفض لهذا المعيار فهي:-

PI > يقبل

PI > Pارفض

PI =. يتم الرفض أو القبول حسب حاجة الثركة لذلك الاسنثار 
أكبر من واحد. وهذا يساعد على انتقاء البدائل الاستثمارية PI ستكون موجبة في حالة NPVوعليه فإن

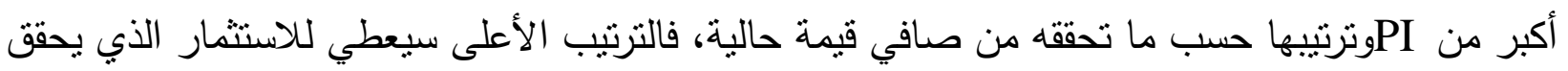
بين مجموعة البدائل الاستثمارية الأخرى. r-r - تحليل قرارات استبدال الموجودات الإنتاجية باستخدام الأساليب الكمية

تعد قرارات استبدال تقنيات الإنتاج وتحديد طرائق المفاضلة بينها من أهم القرارات التي نواجه الثركات. إذ نسعى إدارة العطليات إلى استخدام بعض الأساليب والطرائق الكمية لمعالجة حالة المخاطرة وعدم التأكد المتضمنة في بيئتها، وذلك لمساعدتها في انخاذ القرارات وتحليل مجموعة من البدائل واختيار أنسبها وبالإضافة إلى معايير تحليل الاستثمار المالي التي عرضت آنفاً، فهناك مجموعة أخرى من الأساليب الكمية

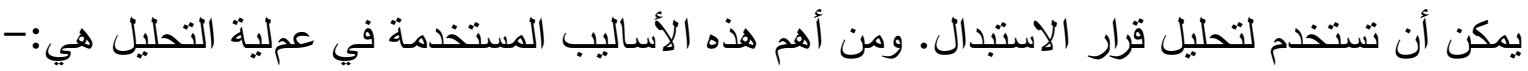

\begin{tabular}{|c|c|}
\hline Replacement Models & نماذج الاستبدال \\
\hline Break Even Analysis & تحليل نقطة التعادل \\
\hline Queuing Theory & نظربة صفوف الانتظار \\
\hline Markov Chains & سلاس - س \\
\hline
\end{tabular}

\section{隹 replacement Models}

تعالج نماذج الاستبدال حالات متعددة ومختلفة وذلك للمقارنة والمفاضلة بين البدائل المختلفة بغية

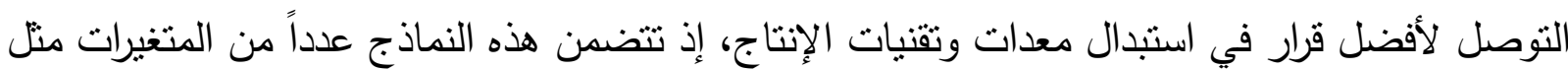

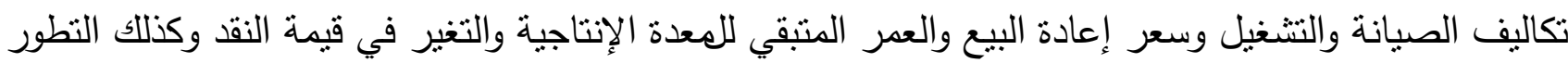
التقني للمكائن والمعدات وغيرها من ال متغيرات التي نواجه الهنظمات ، مما يستلزم تحديدها وتطبيق النموذج

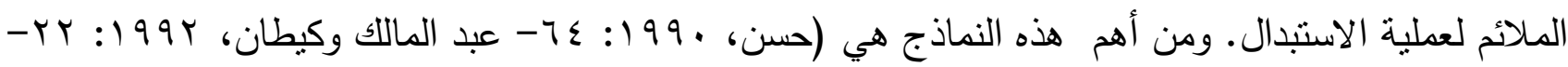
.$(r+1)$

\section{First Year Usage Cost Method}

بموجب هذه الطريقة تحسب تكاليف تشغيل وصبانة الماكنة أو المعدة للسنة الأولى فقط ولكل بديل من البدائل المتاحة للاستبدال، ومن ثم تتم عملية المفاضلة بين النتائج ويتخذ القرار على ضوء ذلك. إن هذه الطريقة تتميز بسهولتها وبساطتها لعدم احتباجها لبيانات متعددة لغرض حسابها كما أن نتائج حساباتها تتسم بالدقة لمحدودية مديات المعلومات واقتصارها على سنة واحدة وتحسب كلفة الاستخدام طبقاً للقاعدة الآتية:C. $R=(p . L) C R F+L I$

$$
\frac{I(1-I)^{N}}{(1+I)^{N}-1} \quad \text { C.R.F. }=
$$

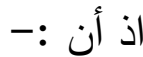

C. R. F =. معامل رأس المال المستثمر 


$$
\text { P = التكاليف الأولية للماكنة }
$$

L القيمة المتبقية نهاية عمر الماكنة.

$$
\begin{aligned}
& \text { p = معامل الفائدة. } \\
& \text { ع = عدد السنوات }
\end{aligned}
$$

\section{Average Life Cost Method}

تتم المقارنة بموجب هذه الطريقة على أساس متوسط تكاليف التتغيل والصيانة لكل بديل ولكل السنوات المقدرة لعمر الماكنة أو المعدة، ويحسب هذا المتوسط بموجب القاعدة الآتية:-

$$
\mathrm{AAC}=(\mathrm{D}+\mathrm{CC}+\mathrm{I}) \mathrm{N}
$$

اذ أن:

AAC معدل الكلفة السنوية.

الاندثار . = الانينة

CC.تكاليف التشغيل =

I = معدل الفائدة على رأس المال المستثمر

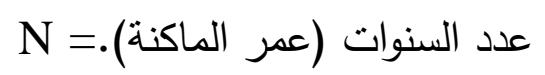

MAPI Method

وضعت هيئة مابي ،وهي جهة استثارية لمنظمة منتجي المكائن والمعدات الأمريكية ، أسلوب يعتمد على الربح المتحقق من جراء عملية Machinery and Allied Products Institute) الاستبدال. فقد وضعت طريقة لإظهار العوائد من جراء شراء ماكنة أو معدة جديدة للسنة التي تلي شراء الماكنة

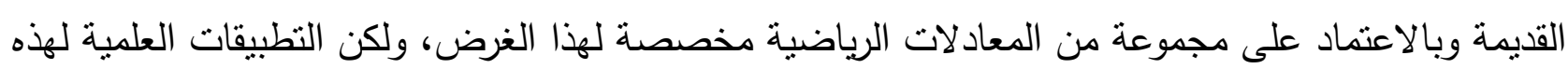
المعادلات اتسمت بصعوبة ترجمتها وتطبيقها في الحياة العملية.

تعتمد هذه الطريقة على تحليل التكاليف المباشرة وغير المباشرة (لمدة عام ) بين الاستمرار في استخدام

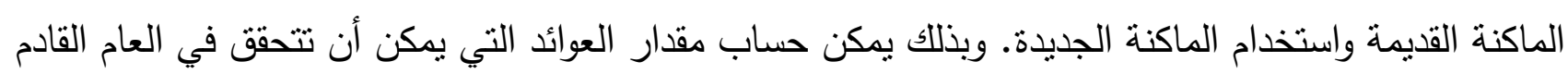
لو استبدلت الماكنة القديمة بأخرى حديثة. هذه الطريقة تختلف عن الطرائق السابقة لأنها لا تعتم د على متوسط التكاليف السنوية أو تكاليف الماكنة خلال فترة انتغالها بشكل رئيسي، فهي تعتمد على مقدار الربح المتحقق في السنة القادمة.

\section{Thabulation Method}

تعد الطريقة الجدولية من أفضل وأسهل الطرائق لتحديد العمر الاقتصادي المثالي لكل ما كنة أو معدة إنتاجية والذي على ضوئه يتخذ قرار الاستبدال من عدمه.

وتعتمد هذه الطريقة على العوامل الآتية:"- 
أ- تكاليف النتغيل والصيانة السنوية.

ب- تكاليف التشغيل والصيانة التزاكمية.

ج- تكاليف النقص في رأس المال المستثمر (سعر الثراء - سعر إعادة البيع).

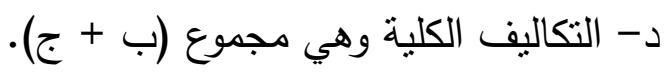

(تكاليف التشغيل والصيانة التراكمية + تكاليف النقص في رأس المال المستثر ).

هـ - عمر الاستخدام للماكنة.

و - متوسط الكلفة السنوية (د/س).

إن الهدف الرئيسي من هذه الطريقة يمثل بيان أهمية اعتماد نموذج يتسم بسهولة حسابه وتطبيقه دون

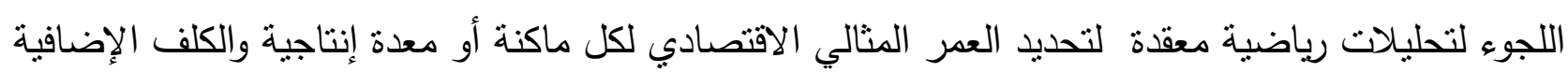
المترتبة على الاستمرار باستخدام هذه المكائن والمعدات بعد هذا العمر ، الأمر الذي يتيح للإدارة خيار الاستبدال أو تحمل كلف التتغيل والصبانة المتزايدة في حالة عدم الاستبدال.

أن نماذج الاستبدال تقوم على افتراض ثبات العمر وإمكانية حسابه بدقة وكما هو موضح في الطرائق

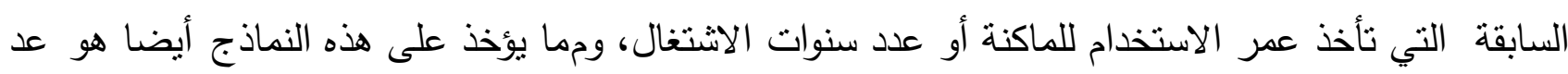

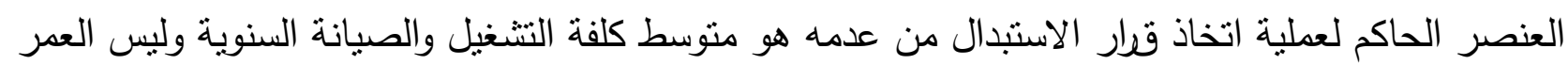

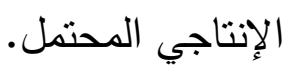

\section{}

يعتبر تحليل نقطة التعادل أداة تحليلية مهمة لفهم العلاقات بين الكلف الثابتة والكلف المتغيهة والعوائد، فهي مؤشر جيد لتحديد النقطة التي يغطي فيها حجم العوائد حجم الكلف الكلية، فالثركة تصبح في حالة " تعادل

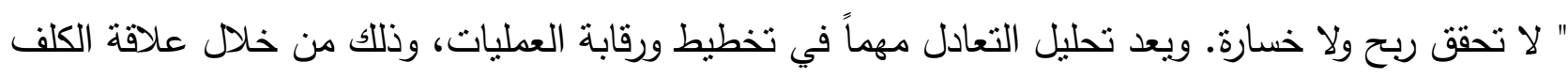
(Westonet al.,

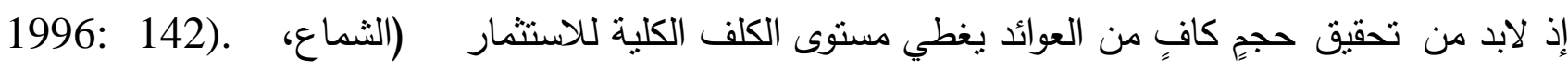

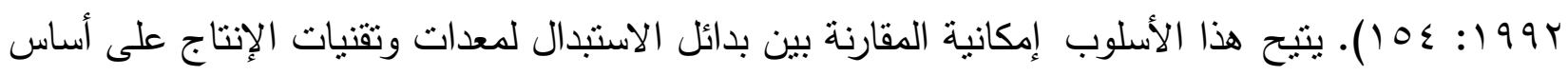

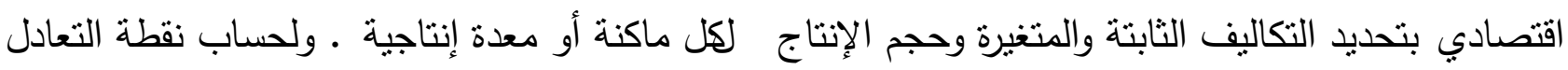

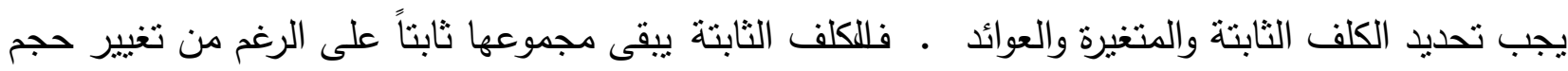
ـ أما الكلف المتغيرة فهي (Moore and Hendrick, 1980:20) الإنتاج وذللك ضمن حدود الطاقة المتاحة تللك الكلف التي يتغير مجموعها بتغير حجم الإنتاج إلا أن الكلفة المتغيرة للوحدة الواحدة تنقى ثابتة كما هي (الثماع، 1991 19 100). إن تحليل نقطة التعادل يمكن أن يطبق في مجالات واسعة، إذ يعدُ دليلّ لمدراء العمليات في اتخاذ قراراتهم، ومنها قرارات الاستبدال.

ولتحليل نقطة التعادل واستخراج حجم التعادل يتم استخدام مجموعة من المعادلات الجبرية للوصول إلى في حجم التعادل المثالي عن طريق تطبيق مجموعة من الخطوات التتابعية. 


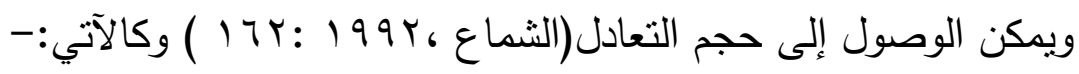

حجم التعادل بالوحدات

$$
(\mathrm{Y}-1 \cdot) \quad\left(\frac{F c}{P-V C}\right) \quad \mathrm{Q}=
$$

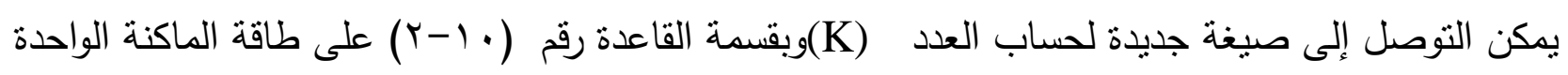
اللازم من المعدات الإنتاجية:- المكية الماحة

$$
\begin{aligned}
& Q=\left(\frac{F C}{P-V C}\right) \div K \\
& Q=\frac{F c}{P-V C} \times \frac{1}{K}
\end{aligned}
$$

وتنساوي أيضا

$$
\frac{F C}{(P-V C) K}=\mathrm{Q}
$$

عدد الآلات والمكائن المتحقق عن نقطة التعادل. ولعبح

\section{المنليا}

يقدم تحليل التعادل العديد من المزايا تستقيد منها إدارة الثركة في اتخاذ قراراتها المهمة:-

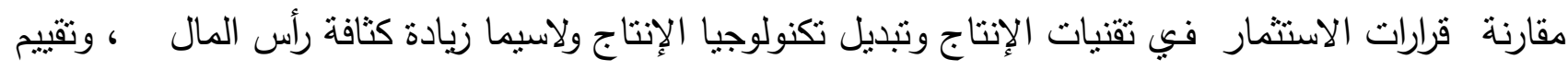

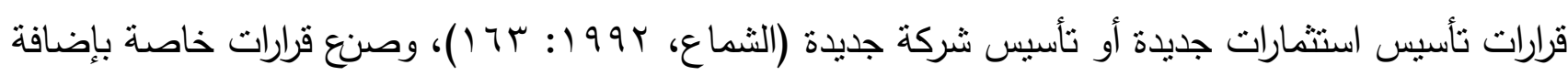

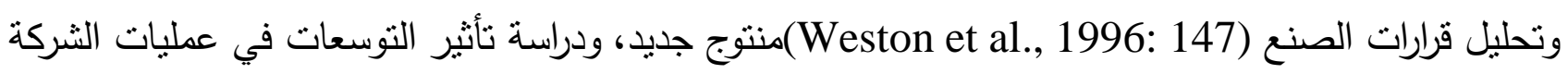
“أو الثراء Make-buy " (Moore and Hendrick, 1980: 26-27).

\section{العبيوي المبراء}

على الرغم من المزايا التي تحملها نقطة التعادل إلا أنها في الوقت نفسه تثمل بعض العيوب والتي من أهمها

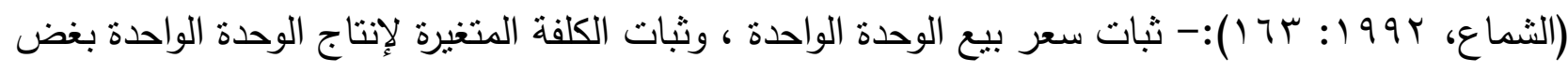

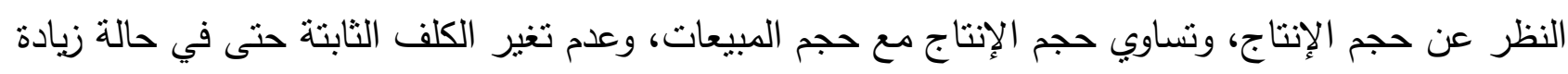
الاستثمار في التقنيات الجديدة.

يمكن اعتبار نقطة التعادل دليلاً جيداً لتحليل قرارات الاستبدال عن طريق الدقارنة والدفاضلة بين عمليات

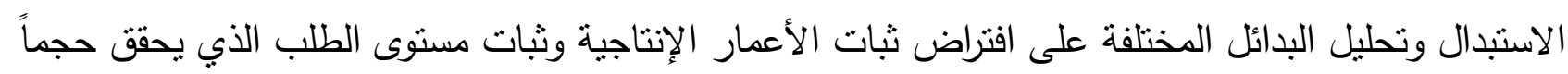
ثابتاً من المبيعات والكلف.

\section{-r Queuing Theory}

اتسعت مجالات استخدام الأساليب الكمبة في السنين الأخيرة وبشكل كبير في معظم نواحي الحياة

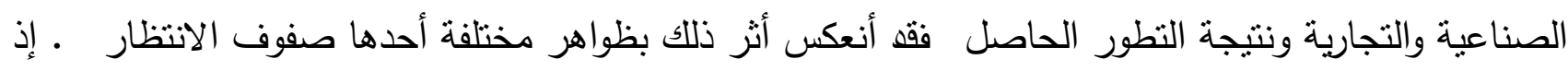


يمكن تطبيقها في مجالات الحياة المتعددة والتي تتميز بوقوف عدد من الوحدات طلباً لخدمة ما من نظام معين كما في المصرف أو عيادة الطبيب. فهي تهنم بدراسة النظام الحالي لأجل تحسينه أو تغييره أو اقتراح نظام جديد بدلاً عنه مع مراعاة الكلفة.

أن أولى محاولات التعبير عن ظاهرة صفوف الانتظار ابتأت بأعمال مهندس الهواتف الدنماركي الجنسية Erlang عام 9 ( 9 (، عندما قام بدراسة مشكلة الاختتاق في تلقي المكالمات الهاتقية إذ كان هدف الدراسة تقليل • وتظورت فيما بعد نظرية صفوف الانتظار (Russell and Taylor ш, 1998: 745 من انتظار المكالمات نتيجة للجهود التي قام بها عددٌ من الباحثين .وتم تعريف نظرية صفوف الانتظار على أنها " دراسة لخطوط ، لغرض تحليل طوابير وتصميم نظم تساعد المنظمة على Queues أو الطوابير Waiting Linesالانتظار • وقد استخدمت نماذج (Lawerence and Pasternack, 1998:532)تحقيق أداء مثالي وفق معايير محدة صفوف الانتظار في اتخاذ قرارات الاستبدال للموجودات الإنتاجية وذلك باعتبار الموجودات الإنتاجية وحدات (Budnick et al,: 1977)

والتي تمثل Arrivals إن العناصر الأساسية لصفوف الانتظار تتمثل بالوحدات القادمة أ و الواصلة مدخلات النظام وقاعدة صف الانتظار Service (Render and Heizer, 2001: • ولإيجاد الحلول المتعلقة بمشاكل الانتظار يتم وضع عدد من الافتراضات للنظام موضوع البحث، ممثلة (805 بمجموعة من المعادلات الرياضية الت ي تعكس حركة ومتغيرات النظام من أجل الحصول على حلول مؤكدة للمشكلة. وهذا ما يطلق عليه نظرية صفوف الانتظار في حين أن معظم حالات صفوف الانتظار الحقيقية تتميز بالتعقيد بحيث لا تحقق كثيراً من الافتراضات التي تستتد عليها النظرية، مما يجعل الأنموذج عبارة عن تمثنيل

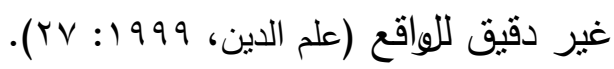

\section{- rMarkov Chain Analysis}

تعد سلاسل ماركوف وسيلة يتم من خلالها تحليل التغيرات الحالية لمتغير عشوائي معين للتنبؤ بالتغيرات • ولغرض A-Markovالمستقبلية لذلك المتغير • وقد أطلق عليها سلاسل ماركوف نسبة إلى العالم الرياضي التعرف على تحليل ماركوف لابد من استخدام الطرق التحليلية والاستتاجات الرياضية لتحديد خصائصها. ينظر إلى العمليات العشوائية على أنها سلسلة من الحالات التي تمر بها ظاهرة ما (كاستبدال تقنيات الإنتاج مثنلاً) خلال فترة زمنية معبنة. وبعبارة أخرى يقال لأي ظاهرة حقيقية تجرى في حيز معين (كالزمن مثنلاً) بأنها عملية عشوائية، إذا كانت حالات تللك الظاهرة في أي جزء من حيزها (في أي وقت كان) تمثل نتائج تجربة تخضع لقوانين الاحتمالات (الربيعي وعبد، . . . ب: 1). أو أنها مجموعة Random Experiment عشوائية

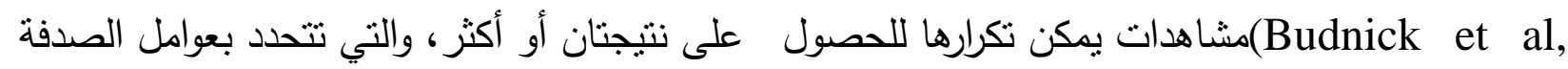
1977: 582) ، 
تعرف عملية ماركوف على أنها نوع محدد من العمليات العشوائية وهي من أكثر العمليات استخداماً

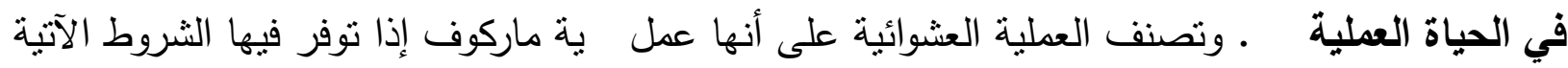
(Shamblin and Stevens, 1974: 53)-:

1- معرفة الحالة الحالية (أو الأقرب إلى الحالة الحالية) لأحد المتغيرات.

r- بموعة النتائج المكنة تكون منتهية.

r- الاحتمالات مستقرة وثابتة خلا الوقت.

تعرف سلاسل ماركوف بأنها عملية عشوائية تتصف بالخواص الآتية:-

$$
\text { Miscrete State Space }
$$

rOne-Step Transition Probability

وتمثل سلاسل ماركوف أحد أصناف عمليات ماركوف والتي عرفت بتطبيقاتها الواسعة في مجال اتخاذ

واثقرارات ومنها تحليل مشاكل الاستبدال وسياسات الصيانة Budnick et al, 1977: 589).

تعالج العطليات العشوائية المشاكل التي تتصف بعدم التأكد البيئي، وهذا ما جعل النماذج العشوائية أكثر

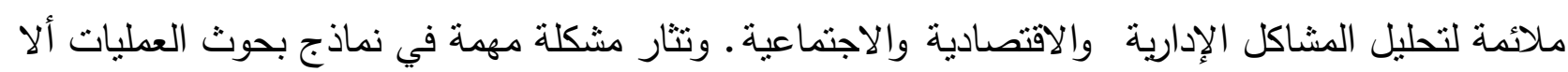
وهي مقدار الفجوة بين النموذج النظري والواقع العملي، وهذه المشكلة يتم تقويمها من خلال عدة اختبارات متمثنة

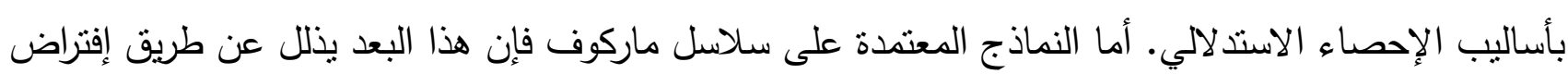

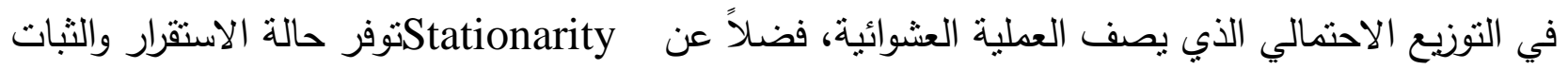

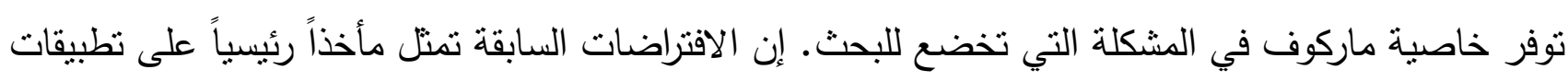
سلاسل ماركوف.

\section{المبحث الثالث \\ منهجية البحث}

\section{r-}

يحّد قرار استبدال معدات وتقنيات الإنتاج وكيفية التخطيط لتوفير منطلبانها النقدية من أهم القرارات التي

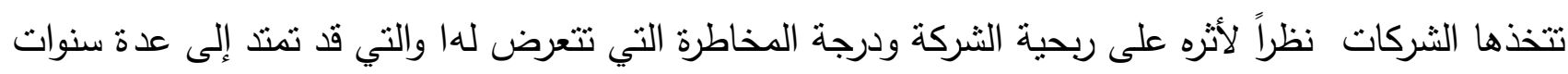

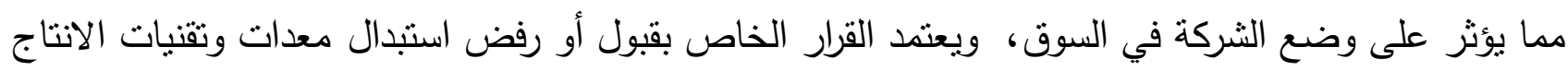

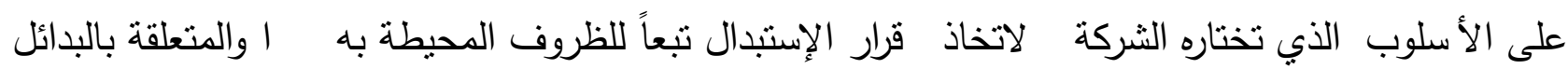

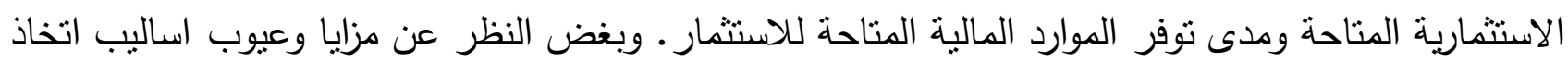
قرار الاستبدال الدذكورة سابقاً، يلاحظ أن تلك الأساليب تفترض ثبات الأعمار الإنتاجية (عمر إنتاجي مخطط)

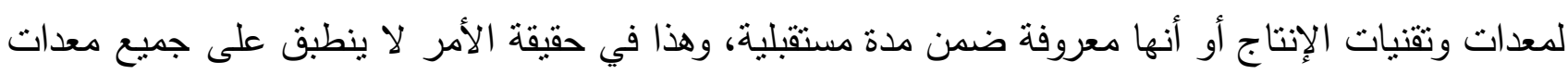




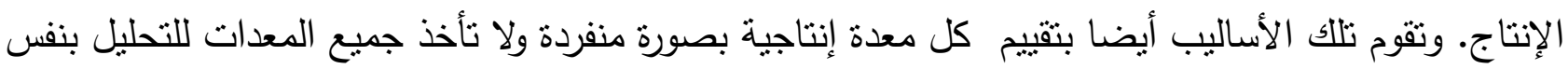

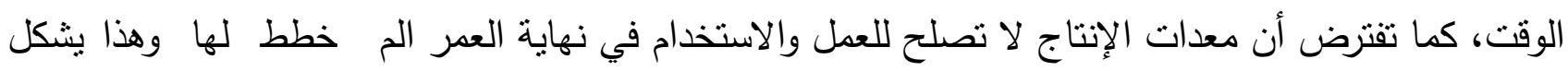

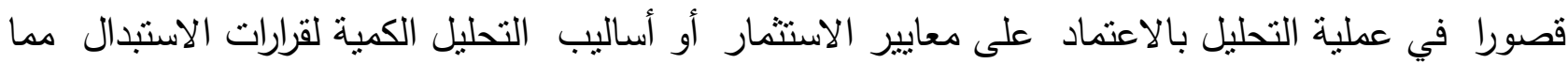

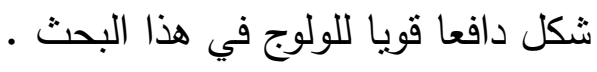

\section{r-r}

$$
\text { تتصب أهداف البحث على الآتي:- }
$$

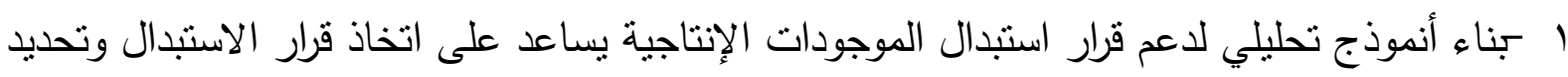

$$
\begin{aligned}
& \text { العدد المحتمل من الموجودات الواجب التهيؤ لثرائها باستخدام المحاكاة. } \\
& \text { r - - عرض وتقويم الأساليب الكمية والمالية المتاحة لاتخاذ قرارات الاستبدال. }
\end{aligned}
$$

r تززويد متخذ القرار بقاعدة علمية للتفاوض بثأن الاحتياجات المالية السنوية لنشراء الموجودات الإنتاجية

$$
\text { سواء كان تمويلا مركزيا أوذاتيا . }
$$

\section{ب- - r أهمية البحث}

$$
\text { تتحدد أهمية البحث بالآتي: }
$$

1 - محدودية الدراسات التي تتاولت تحليل قرارات الاستبدال باستخدام أسلوب تحليلي متمنل بالمحاكاة، ولا سيما

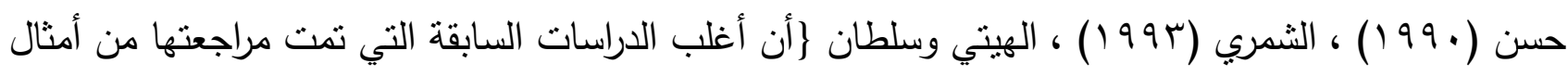
، (199)D’aversa and Shapiro, 1987 ، Nguyen and Murthy, 1984 ، Whitaker, 1984 ،

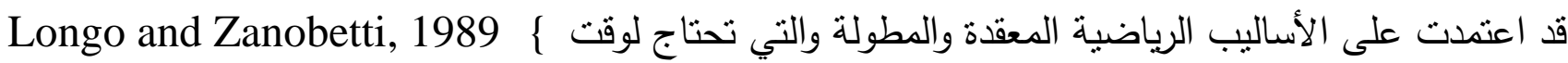

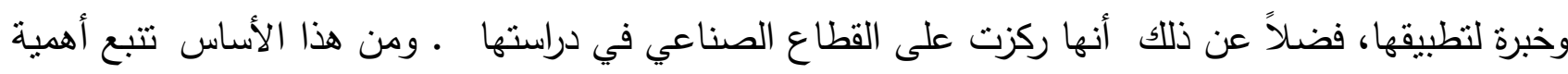

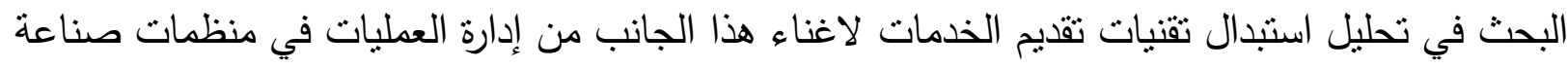

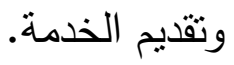

r- تمثل الخدمات أحد المرتكزات الأساسية للنطور الحضاري وإن التخطيط لهذا القطاع الحيوي بشكل علمي مدروس سوف يعزز من دوره وموقعه في عملية التتمية الإقتصادية فضلاً عن تمكين إلا دارة من تحقيق أهدافها الإستراتيجية وهو ما بعطي أهمية متميزة للبحث.

\section{ب- ع- فرضبة البحث}

بما إن معايير الاستثمار المالي والأساليب الكمية التي استعرضت آنفا تفترض ثبات العمر الإنتاجي للموجودات الإنتاجية ، فان هذا البحث سيحاول اختبار الفرضية الآتية:-

(لا يرتبط الوسط الحسابي لعدد مرات استبدال الموجودات الإنتاجية التي ينبغي التهيؤ لاستبدالها ارتباطاً معنوياً بالعمر الإنتاجي المخطط لتبك الموجودات)). 


\section{ب-ه - أسالبب البحث والتحليل}

أستتد البحث في تحليل البيانات والتوصل إلى النتائج المطلوبة على استخدام بعض الأساليب الكمية وكالآتي:-

Simulation لتحليل مشكلة البحث، حيث تم الاعتماد عليها في بناء الأنموذج واستخراج النتائج وإجراء تجارب للحصول على بيانات عن الاستبدال واستخدام تلك البيانات في اختبار فرضية البحث.

ثانيا- برمجية اكسل - حيث استخدمت في بناء نموذج دعم قرار الاستبدال ونشغيله.

ثالثا - الأساليب الإحصائية- استخدم معامل الإزتباط البسيط Simple Correlation Coefficient لتهيئة البيانات اللازمة لاختبار فرضية البحث، كما استخدم اختبار t لهذا الغرض.

\section{المبحث الرابع}

\section{تصميم نموذج دعم قرار الاستبدال}

واحدة من أساليب بحوث العمليات الفعالة والمفيدة، إذ تستخدم في الحالات Simulation تعد المحاكاة التي لا يمكن التجريب فيها على النظام الحقيقي لصعوبة التطبيق ، لهذا تعد من أكثر طرائق التحليل الكمي استخداماً وعلى الرغم من ذلك فهي لا نشكل الحل الوحيد لكل مشاكل إدارة العمليات ولكنها تعتبر من أكثر • وتعرف المحاكاة على أنها:-Flexible-طرائق التحليل الكمي مرونة

"(Heizer and Render, 2001: T108). محاولة لنسخ خصائص ومظاهر وصفات النظام الحقيقي" :

وسيتم في هذا المبحث بناء نموذج محاكاة من نوع مونت كارلو يعتمد على البيانات التاريخية للاستبدال وتوزبعه التجريبي ، لان عمليات الاستبدال السابقة التي يعكسها التوزبع التجريبي تضم جميع العوامل (مالية ، أو تكنولوجية ، أو حوادث العمل، او الطبيعية وغيرها) التي استدعت الاستبدال في السابق . وقد استخدم الباحثون والمتكونة من الخطوات الاتية :- تعريف Render and Heizer منهجية المحاكاة التي اشار اليها كل من

المشكلة ، جمع البيانات ، بناء النموذج ، تصميم وتنفيذ العملية ، المصداقية الخارجية والمصداقية الداخلية • ونظرا لمحدودية عدد الصفحات (Heizer and Render, 2001: T108)للمحاكاة ، عرض وتقبيم نتائج المحاكاة المسموح بها للنشر فسوف لا يتطرق الباحثون الى تفاصيل منهجية المحاكاة.

وينكون النظام الذي اعده الباحثون من عدة صحائف تمثل "صحيفة مدخلات النظام " ، "صحيفة المعالجة" ، " "صحيفة المحاكاة" ، "صحيفة المستلزمات المالية".

وكما هو معروف فان أي نظام يتكون من العناصر الآتية :- المدخلات ، المعالجة ، المخرجات ، والتخذية العكسية وفيما يلي نقدم وصفا للعناصر التي سيضمها النظام.

$$
\text { ا. المدخلات :- - تتضمن مدخلات نظام دعم قرار الاستبدال الاتي:- }
$$

توزيع تكراري تجريبي يمثل عملية استبدال الموجودات الإنتاجية المنتابهة في الماضي ولإغراض عرض وتتغيل النظام فقد اعتمد الباحثون على مثال افتراضي تطبيقي Case Example وتشمل 
الدخلات بيانات تاريخية عن عمليات الاستبدال لباصات نقل المسافرين في إحدى شرك ات النقل

$$
\text { البري للمسافرين، وكما موضح في الجدول (ع - (1). }
$$

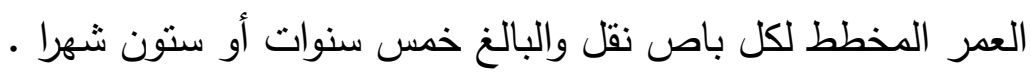

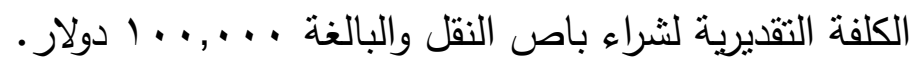

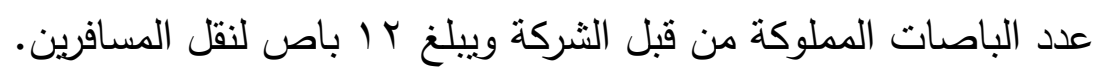

عدد الباصات في الخدمة ويساوي أيضا ب ا باص.

هعر الفائدة السائد في السوق والذي سيستخدم لحساب القيمة الحالية للمتطلبات المالية اللازمة في باصي

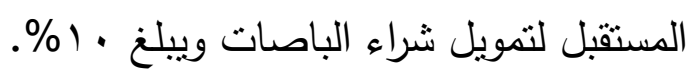

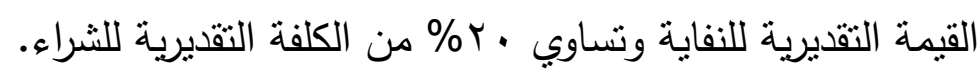

إذ يمكن للمستقيد إدخال Excelوالثكل (ع - () يوضح هيئة مدخلات النظام وهي معدة بموجب برمجية بياناته في الحقول المضللة فقط.

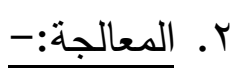

تمثل محاكاة مونت كارلو جوهر المعالجات التي يقوم بها النظام ، وفيما يلي وصف مختصر

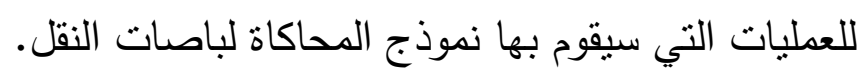

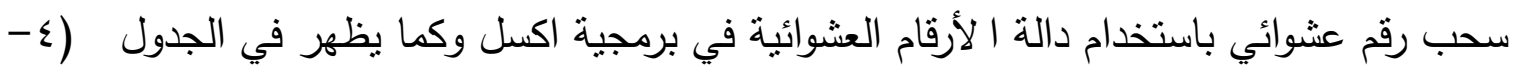

باستخدام دالة V-lookup في برمجية اكسل والتوزيع التجريبي والرقم العشوائي الأول للباص الأول

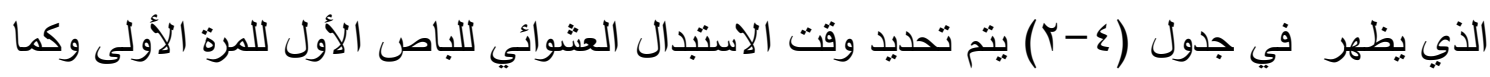

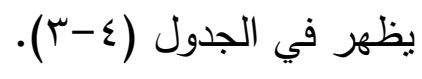

باستخدام دالة V-lookup في برمجية اكسل والتوزيع التجريبي والرقم العشوائي الثاني للباص الأول

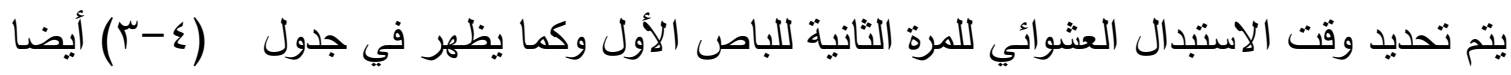
وهكذا لغاية بلوغ العمر المخطط او تجاوزه.

يحسب العمر التراكمي للباص وذللك بلضضافة وقت الاستبدال الأول إلى وقت الاستبدال الثاني ووقت

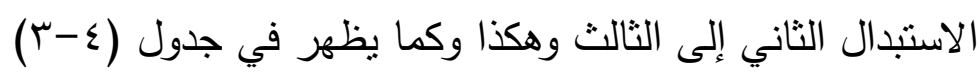

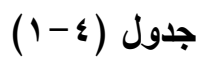

\begin{tabular}{|c|c|c|}
\hline ألاحتمال & ألاحتمال & عمر الباصن \\
\hline$\because .0$ & $\because .0$ & IT \\
\hline .10 & .1 & 10 \\
\hline . ro & $\theta^{r} \cdot$ & 11 \\
\hline 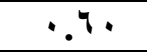 & $\therefore$. ro & $r$ \\
\hline$\because 98$ & ध & $Y \varepsilon$ \\
\hline
\end{tabular}

التوزيع الاحتمالي التجريبي لعملياتاستبدال باصات النقل 


\begin{tabular}{|c|c|c|}
\hline $.9 \varepsilon$ & $\ddots \cdot \varepsilon$ & $r v$ \\
\hline $1 . \cdots$ & $\ddots \cdot q$ & $r$ \\
\hline
\end{tabular}

المصدر: من إعداد الباحثون

صحيفة مدخلات النظام

التوزيع التجريبي لاعمار باصات النقل بالاشهر

\begin{tabular}{|c|}
\hline عدد الباصات المملوكة \\
\hline 12 \\
\hline عدد الباصـات في الخدمة \\
\hline 12 \\
\hline العمر المخطط بالسنو ات \\
\hline 5 \\
\hline العمر المخطط بالاشهر \\
\hline 60 \\
\hline الكلفة التقديرية \$ \\
\hline 100000 \\
\hline سعر الفائدة \\
\hline $10 \%$ \\
\hline
\end{tabular}

\begin{tabular}{|c|c|}
\hline 1 - عمر الباص بالاشهر \\
\hline 0 & 12 \\
\hline 0.05 & 15 \\
\hline 0.15 & 18 \\
\hline 0.35 & 21 \\
\hline 0.6 & 24 \\
\hline 0.9 & 27 \\
\hline 0.94 & 30 \\
\hline
\end{tabular}

القيمة التقديرية للنفاية = =

ملاحظة يرجى ادخال البيانات في الحقول المضللة فقط

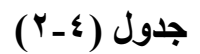

محاكـاة عمليـة الاسـتبلدال

سحب لارقام العثوائية

\begin{tabular}{|c|c|c|c|c|}
\hline RN\#4 & RN\#3 & RN\#2 & *RN\#1 & رق \\
\hline $\mathbf{0 . 4 6}$ & $\mathbf{0 . 1 9}$ & $\mathbf{0 . 5 6}$ & $\mathbf{0 . 7 6}$ & $\mathbf{1}$ \\
\hline $\mathbf{0 . 2 4}$ & $\mathbf{0 . 1 9}$ & $\mathbf{0 . 2 6}$ & $\mathbf{0 . 2 1}$ & $\mathbf{2}$ \\
\hline $\mathbf{0 . 2 0}$ & $\mathbf{0 . 2 5}$ & $\mathbf{0 . 4 6}$ & $\mathbf{0 . 1 6}$ & $\mathbf{3}$ \\
\hline $\mathbf{0 . 7 6}$ & $\mathbf{0 . 9 3}$ & $\mathbf{0 . 3 6}$ & $\mathbf{0 . 8 9}$ & $\mathbf{4}$ \\
\hline $\mathbf{0 . 8 4}$ & $\mathbf{0 . 8 8}$ & $\mathbf{0 . 5 3}$ & $\mathbf{0 . 9 3}$ & $\mathbf{5}$ \\
\hline $\mathbf{0 . 0 9}$ & $\mathbf{0 . 9 2}$ & $\mathbf{0 . 9 6}$ & $\mathbf{0 . 3 4}$ & $\mathbf{6}$ \\
\hline $\mathbf{0 . 9 2}$ & $\mathbf{0 . 2 0}$ & $\mathbf{0 . 1 3}$ & $\mathbf{0 . 3 2}$ & $\mathbf{7}$ \\
\hline $\mathbf{0 . 5 9}$ & $\mathbf{0 . 9 6}$ & $\mathbf{0 . 8 4}$ & $\mathbf{0 . 0 4}$ & $\mathbf{8}$ \\
\hline $\mathbf{0 . 7 7}$ & $\mathbf{0 . 0 5}$ & $\mathbf{0 . 9 9}$ & $\mathbf{0 . 4 8}$ & $\mathbf{9}$ \\
\hline $\mathbf{0 . 3 6}$ & $\mathbf{0 . 6 1}$ & $\mathbf{0 . 9 6}$ & $\mathbf{0 . 4 1}$ & $\mathbf{1 0}$ \\
\hline $\mathbf{0 . 9 1}$ & $\mathbf{0 . 1 1}$ & $\mathbf{0 . 6 2}$ & $\mathbf{0 . 1 8}$ & 11 \\
\hline $\mathbf{0 . 2 1}$ & $\mathbf{0 . 0 8}$ & $\mathbf{0 . 0 7}$ & $\mathbf{0 . 2 4}$ & $\mathbf{1 2}$ \\
\hline
\end{tabular}

RN\#1 *

جدول (r-ع)

لتوزيع التجريبي الاحتمالي

\begin{tabular}{|c|c|}
\hline عمر الباص & من صفر - المدى 1 \\
\hline 12 & $\mathbf{0}$ \\
\hline 15 & 0.05 \\
\hline 18 & 0.15 \\
\hline 21 & 0.35 \\
\hline 24 & 0.6 \\
\hline 27 & 0.9 \\
\hline 30 & 0.94 \\
\hline
\end{tabular}

الجدول الزمني المحتمل للآستبدال والاعمار التراكمية للباصات بالاشهر

\begin{tabular}{|c|c|c|c|c|}
\hline الاستبدال الرابع & الاستبدال الثالث & الاستبادال الثانى & الاستبدال الاول & رقم الباص \\
\hline $\mathbf{0}$ & 63 & 42 & 21 & 1 \\
\hline 0 & 60 & 42 & 21 & 2 \\
\hline $\mathbf{0}$ & 78 & 48 & 24 & 3 \\
\hline $\mathbf{0}$ & 78 & 48 & 24 & 4 \\
\hline
\end{tabular}




\begin{tabular}{|c|c|c|c|c|}
$\mathbf{0}$ & $\mathbf{6 6}$ & $\mathbf{4 2}$ & $\mathbf{2 1}$ & $\mathbf{5}$ \\
\hline $\mathbf{0}$ & $\mathbf{6 0}$ & $\mathbf{3 6}$ & $\mathbf{1 8}$ & $\mathbf{6}$ \\
\hline $\mathbf{7 5}$ & $\mathbf{5 4}$ & $\mathbf{3 6}$ & $\mathbf{1 8}$ & $\mathbf{7}$ \\
\hline $\mathbf{0}$ & $\mathbf{6 6}$ & $\mathbf{4 8}$ & $\mathbf{2 4}$ & $\mathbf{8}$ \\
\hline $\mathbf{0}$ & $\mathbf{7 2}$ & $\mathbf{4 8}$ & $\mathbf{2 4}$ & $\mathbf{9}$ \\
\hline $\mathbf{0}$ & $\mathbf{6 0}$ & $\mathbf{3 0}$ & $\mathbf{1 5}$ & $\mathbf{1 0}$ \\
\hline $\mathbf{6 9}$ & $\mathbf{5 1}$ & $\mathbf{3 0}$ & $\mathbf{1 5}$ & $\mathbf{1 1}$ \\
\hline $\mathbf{0}$ & $\mathbf{8 1}$ & $\mathbf{6 0}$ & $\mathbf{3 0}$ & $\mathbf{1 2}$ \\
\hline
\end{tabular}

مرات الاستبال والعمر المتبقي المحتمل للباص

\begin{tabular}{|c|c|c|}
\hline عدد مرات الاستبدال & المتبقي للباص المحتمل & رقاص \\
\hline 3 & 3 & 1 \\
\hline 3 & $\mathbf{0}$ & 2 \\
\hline 3 & 18 & 3 \\
\hline 3 & 18 & 4 \\
\hline 3 & 6 & 5 \\
\hline 3 & $\mathbf{0}$ & 6 \\
\hline 4 & 15 & 7 \\
\hline 3 & 6 & 8 \\
\hline 3 & 12 & 9 \\
\hline 3 & $\mathbf{0}$ & 10 \\
\hline 4 & 9 & 11 \\
\hline 3 & 21 & 12 \\
\hline 3.2 & 9.0 & ا الوسط الحسابي \\
\hline 0.4 & 7.7 & الانحراف لمعياري \\
\hline
\end{tabular}

عدد الباصات الصالحة بعد انتهاء العمر المخطط = r I ب باص

• تكرر هذه العملية لغاية الثهر ـ وهو العمر المخطط لباصات النقل في هذه الحالة الافتراضية. • حساب العمر المتبقي من الباص إذا كان العدر التراكمي قد تجاوز ـ شهراً وكما يظهر في جدول

تكرر هذه العملية لجميع الباصات .

ويوضح الثكل (ع-r) المخطط المنطقي لعملية المحاكاة.

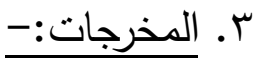

يقدم نظام دعم قرار الاستبدال عدة جداول تمنل مخرجات عملية المحاكاة وعلى شكل صحائف اكسل،

وتتضمن هذه التقارير ما يلي:-

• تقرير بعدد مرات استبدال الباصات المحتملة لكل باص وخلا المدة التخطيطية جدول(ع-ب) . تقرير بالعمر التراكمي المحتمل لباصات النقل جدول (ع-ب).

تقرير بالعمر المحتمل للباص بعد انتهاء العمر المخطط ويحسب بطرح العمر المخطط من آخر

عمر نراكمي لكل باص وكما يظهر في جدول(ع - ؟) . 
• تقرير بالسنوات المحتمل حدوث الاستبدال فيها لكل باص نقل على انفراد وكما ي ظهر في جدول . (0- )

• نتائج محاكاة عملية الاستبدال لثلاثثين مرة وتتضمن الوسط الحسابي لمرات الاستبدال المحتملة لكل

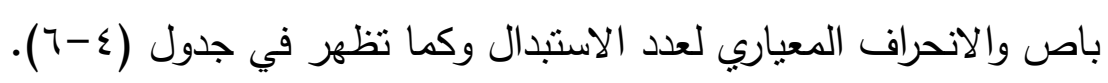

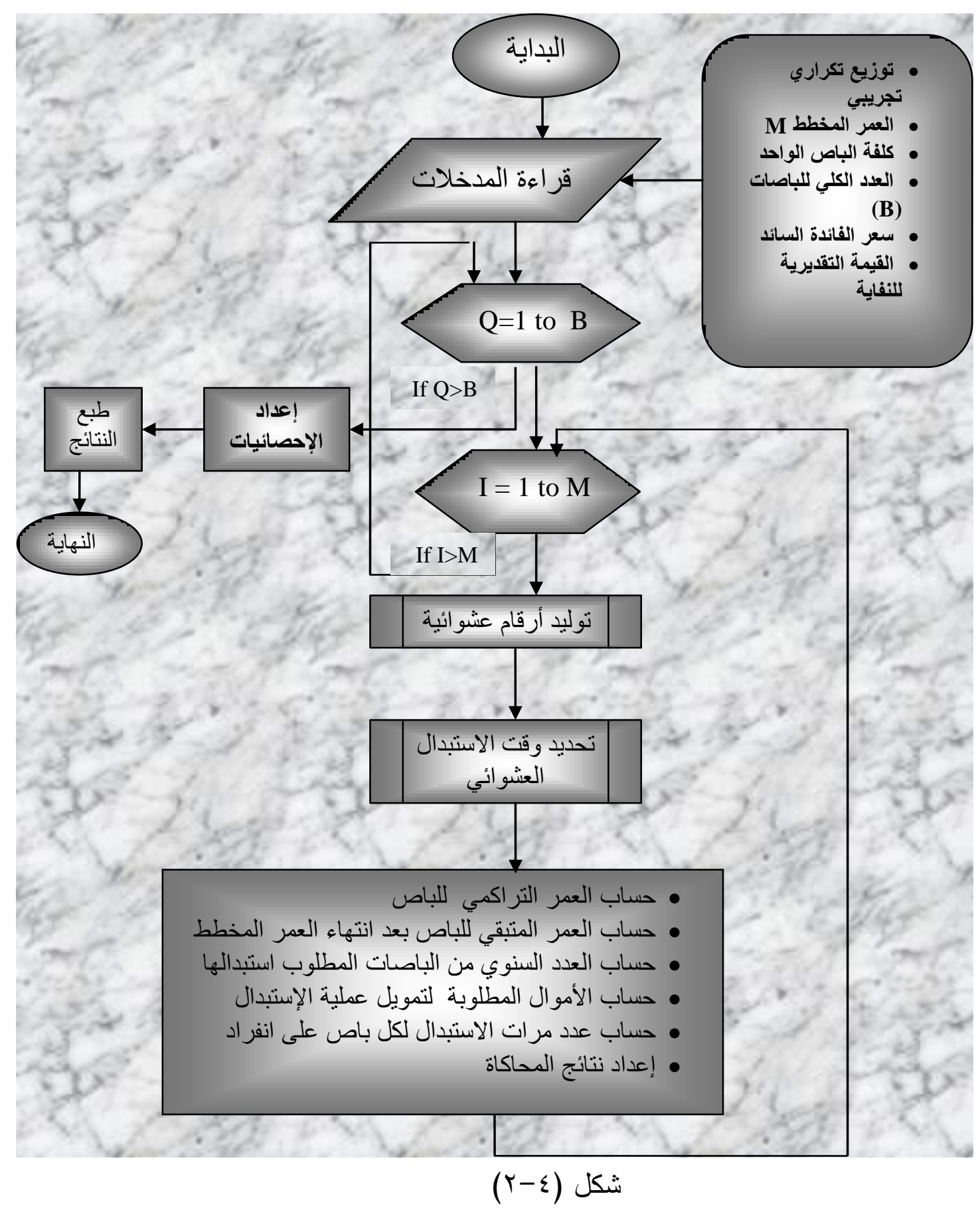

المخطط المنطقي لعملية المحاكاة 


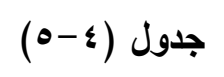

السنوات المحتمل حدوث الاستبدال فيها لكل باص

\begin{tabular}{|c|c|c|c|c|c|c|c|c|c|c|c|c|c|c|c|c|}
\hline & \multicolumn{4}{|c|}{ الباص السادس } & \multicolumn{3}{|c|}{ الباص الخامس } & \multicolumn{2}{|c|}{ الباص الرابع } & \multicolumn{3}{|c|}{ الباص الثاني } & \multicolumn{3}{|c|}{ الباص الاول } & \multirow{2}{*}{ السنة } \\
\hline & & & & & & & & & & & & 1 & & & & \\
\hline & & & & 1 & & & 1 & & 1 & & 1 & & & & 1 & $r$ \\
\hline & & & 1 & & & & & & & & & & & 1 & & $r$ \\
\hline & & & & & & 1 & & 1 & & 1 & & & & & & $\varepsilon$ \\
\hline & & 1 & & & & & & & & & & & 1 & & & 0 \\
\hline مجمو & & ني & U الا & & دي عشر & الد & الباص & س & & الثا & باص & & & ال & & \\
\hline 1 & & & & 1 & & & & & & & & & & & & \\
\hline ir & & & 1 & & & & 1 & & 1 & & & 1 & & & & 1 \\
\hline$\varepsilon$ & & & & & & 1 & & & & & & & & & & \\
\hline 9 & & 1 & & & 1 & & & 1 & & & 1 & & & & & \\
\hline 0 & 1 & & & & & & & & & & & & & & & \\
\hline
\end{tabular}

تقرير بالموارد المالية اللازمة المحتملة سنويا لتمويل عملية الاستبدال مخصومة بالقيمة الحالية للنقود

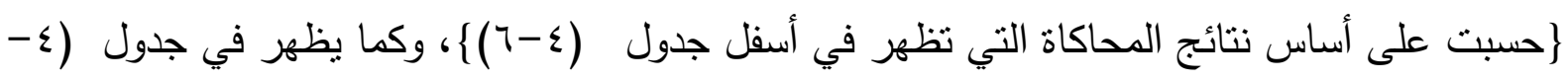

• إعداد مجموعة من الإحصائيات تساعد المدير في اتخاذ قرا ر الاستبدال منل معدل عدد الباصات

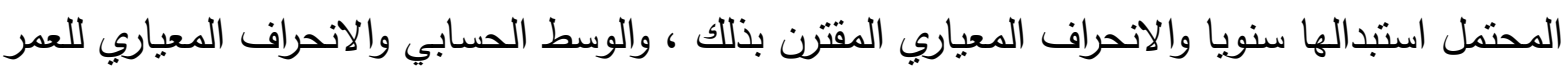

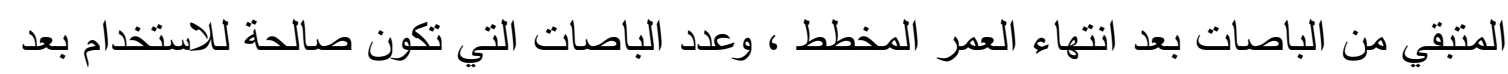

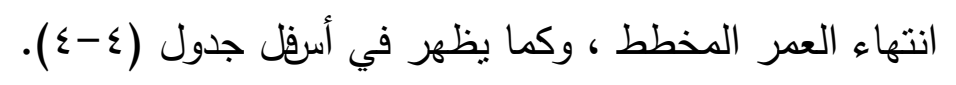
جدول (६- (v)

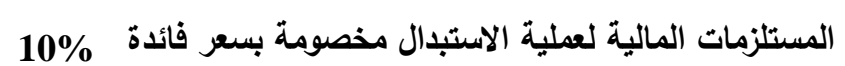

\begin{tabular}{|c|c|c|c|c|c|}
\hline صافي القَّيمة \$ & القيشتر الحالية \$ & 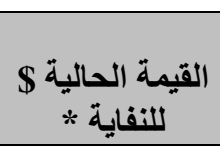 & اللمشتريات غيرة & الاستبدالات عدلد & السنة السنة \\
\hline 4,683 & 12,562 & 7,879 & 43,333 & 0.4 & 1 \\
\hline 689,807 & 862,259 & 172,452 & $1,043,333$ & 10.4 & 2 \\
\hline 309,424 & 395,692 & 86,268 & 526,667 & 5.3 & 3 \\
\hline 328,755 & 444,994 & 116,238 & 716,667 & 7.2 & 4 \\
\hline 311,790 & 407,531 & 95,742 & 596,667 & 6.0 & 5 \\
\hline $1,644,460$ & $2,123,038$ & 478,579 & $2,926,667$ & & | مجموع | \\
\hline
\end{tabular}


ويتمكن المستفيد من اعادة عملية المحاكاة اذا لم يكن مقتتعا بالنتائج او اذا اراد معرفة تأثير تغير بعض المدخلات على نتائج عملية الاستبدال كتغير سعر الفائدة او تغير كلفة الثراء التقديرية او تغير التوزيع التجريبي لعملية الاستبدال . ويتمكن المستفيد ايضا من توسيع او تقليص المخرجات حسب رغبة المستفيد وذلك بإجراء تعديلات بسيطة على النظام المكتوب وفقا لبرمجية اكسل وهذا يتطلب معرفة متواضعة ببرمجية أكسل.

\begin{tabular}{|c|c|c|c|c|c|}
\hline \multicolumn{6}{|c|}{ جدول (ء - 7) } \\
\hline السنة الخامسة & السنة الرابعة & السنة الثالثة & السنة الثانية & السنة الاولى & رقم المحاكاة \\
\hline 5 & 10 & 2 & 11 & 0 & 1 \\
\hline 8 & 9 & 3 & 11 & 0 & 2 \\
\hline 5 & 10 & 2 & 11 & 0 & 3 \\
\hline 7 & 9 & 3 & 11 & 1 & 4 \\
\hline 9 & 3 & 10 & 10 & 0 & 5 \\
\hline 4 & 9 & 4 & 11 & 0 & 6 \\
\hline 4 & 8 & 4 & 9 & 0 & 7 \\
\hline 7 & 6 & 7 & 9 & 0 & 8 \\
\hline 5 & 8 & 3 & 11 & 1 & 9 \\
\hline 7 & 7 & 6 & 10 & 0 & 10 \\
\hline 5 & 8 & 4 & 12 & 0 & 11 \\
\hline 8 & 6 & 6 & 11 & 2 & 12 \\
\hline 6 & 8 & 4 & 11 & 1 & 13 \\
\hline 4 & 7 & 5 & 11 & 0 & 14 \\
\hline 5 & 6 & 6 & 10 & 0 & 15 \\
\hline 6 & 7 & 6 & 11 & 0 & 16 \\
\hline 5 & 7 & 5 & 10 & 1 & 17 \\
\hline 6 & 6 & 6 & 10 & 1 & 18 \\
\hline 5 & 8 & 4 & 11 & 0 & 19 \\
\hline 9 & 5 & 7 & 9 & 1 & 20 \\
\hline 8 & 4 & 9 & 6 & 0 & 21 \\
\hline 7 & 7 & 6 & 10 & 0 & 22 \\
\hline 7 & 5 & 7 & 10 & 1 & 23 \\
\hline 5 & 9 & 4 & 11 & 1 & 24 \\
\hline 4 & 10 & 4 & 12 & 1 & 25 \\
\hline 2 & 9 & 6 & 12 & 0 & 26 \\
\hline 6 & 6 & 7 & 11 & 0 & 27 \\
\hline 7 & 7 & 5 & 10 & 0 & 28 \\
\hline 7 & 5 & 8 & 9 & 1 & 29 \\
\hline 6 & 6 & 5 & 12 & 1 & 30 \\
\hline 179 & 215 & 158 & 313 & 13 & المجموع \\
\hline \multirow[t]{2}{*}{6.0} & 7.2 & 5.3 & 10.4 & 0.4 & الوسط الحسابي \\
\hline & & & & 3.6 & الانحراف المعياري \\
\hline
\end{tabular}

اختبار الفرضية

لاختبار فرضية البحث تم ت محاكاة نموذج دعم قرار الاستبدال لفترات تخطيطية مختلفة لأعمار الباصات إبتداء من: • . فقد حدد ب ب ا باص. وقد حدد النموذج في نهاية كل عملية محاكاة عدد مرات الاستبدال المحتمل حدوثها 
أثناء تلكك المدد وذلك باعتماد نتائج المحاكاة وعلى غرار النتائج المعروضة في الجداول (ع-ب)-(ع-7) ، وقد

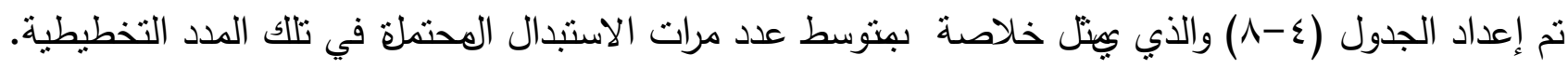

في تحليل البيانات Simple Correlation Coefficient وقد تم استخدام معامل الارتباط البسيط المتغير التابع يمثل (y) وهو المتغير المستقل و (x)أعلاه وعلى اعتبار إن العمر الإنتاجي المخطط للباصات الوسط الحسابي لعدد مرات الاستبدال الهحتملة في المدد المحددة.

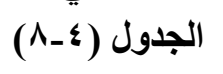

متوسط عدد مرات الاستبدال المحتملة بتغير العمر الإنتاجي المخطط للباص

\begin{tabular}{|c|c|c|c|}
\hline متوسط عدد مرات الاستبدال & العمر المخطط للباصات & العمر المخطط للباصات & ت \\
\hline r & 0 & 7. & 1 \\
\hline 0 & 7 & $V Y$ & $r$ \\
\hline$\varepsilon$ & V & $\Lambda \varepsilon$ & $\Gamma$ \\
\hline 0 & $\Lambda$ & 97 & $\varepsilon$ \\
\hline 0 & 9 & 1.1 & 0 \\
\hline 0 & 1. & $I r$. & 7 \\
\hline
\end{tabular}

ولحساب معامل الارتباط بين العمر الإنتاجي المخطط لتلك المدد ومتوسط عدد مرات الاستبدال فقد تم إعداد الجدول (ع-9) كالآتي:-

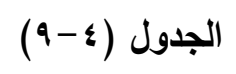

التحليل الإحصائي لحساب معامل الارتباط بين العمر المخطط للباص

\begin{tabular}{|c|c|c|c|c|c|}
\hline No. & xأعمار الباصات المخططة & yمتوسط عدد مرات الاستببال & $\chi^{2}$ & $\chi \mathbf{y}$ & $\mathrm{y}^{2}$ \\
\hline 1 & 0 & $r$ & 25 & 10 & 9 \\
\hline r & 7 & 0 & 36 & $r$. & ro \\
\hline$r$ & $\mathrm{~V}$ & $\varepsilon$ & $\leqslant 9$ & $r \wedge$ & 17 \\
\hline$\varepsilon$ & $\Lambda$ & 0 & $7 \varepsilon$ & $\varepsilon$ & ro \\
\hline 0 & 9 & 0 & $\Lambda 1$ & $\leqslant 0$ & ro \\
\hline \multirow[t]{2}{*}{7} & 1. & 0 & $1 \ldots$ & 0. & ro \\
\hline & «0 & $F V$ & $r 00$ & $r \cdot \Lambda$ & $1 \% 0$ \\
\hline
\end{tabular}

ومتوسط عدد مرات الاستبدال

وبتطبيق معادلة معامل الإرتباط البسيط الآثية:-

$$
\begin{gathered}
r=\frac{n \sum x y-\left(\sum x\right)\left(\sum y\right)}{\sqrt{\left[n \sum x^{2}-\left(\sum x\right)^{2}\right]\left[n \sum y^{2}-\left(\sum y\right)^{2}\right]} \ldots \ldots . . .(4-1)} \\
\quad r=\frac{6(208)-(45)(27)}{\sqrt{\left[6(355)-(45)^{2}\right]\left[6(125)-(27)^{2}\right]}}
\end{gathered}
$$




$$
\text { الارتباط موجب }=\frac{1248-1215}{\sqrt{(105)(21)}}=\frac{33}{46.957}=0.702
$$

وبالإعتماد على الفرضية t سوف نعتمد على اختبار (y) و (x)وللوقوف على معنوية الإرتباط بين

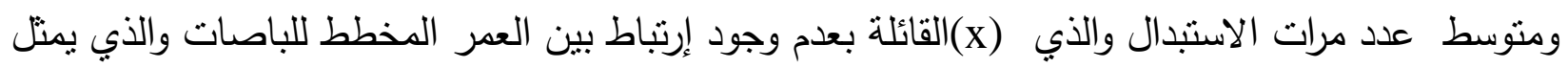

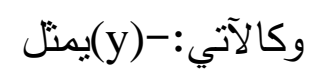

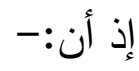

$$
\begin{aligned}
& \mathrm{H}_{0}: \mathrm{P} \neq 0 \\
& \mathrm{H}_{1}: \mathrm{P}=0 \\
& t=r \sqrt{\frac{n-2}{1-3}}
\end{aligned}
$$

$\mathrm{t}=0.702 \times \sqrt{\frac{6-2}{1-(0.702)^{2}}}$

$\mathrm{t}=0.702(2.808)$

$\mathrm{t}=1.971$

المحسوبة مع القيمة النظرية وعند درجة حرية ؛ ومستوى معنوية ه .. . يتضح أن tوعند مقارنة قيمة

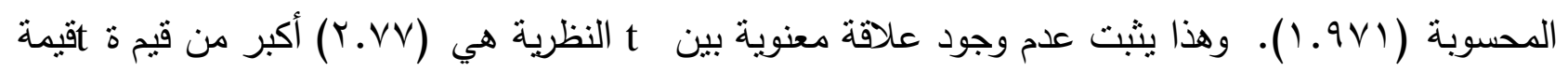
متوسط العمر الإنتاجي للباصات ومتوسط عدد مرات الاستبدال المحتملة مقابل عدد الباصات التي ينبغي التهيؤ لشرائها. مما يؤيد صحة الفرضية بمعنى آخر إن العمر التخطيطي مهما تغير لن يؤثر على مصير الباص.

\section{خاثقة}

1 - قدم الباحثّن في هذا البحث اداة جديدة لتحليل مشكلة الاستبدال بوصفها مشكلة دينامية تأخذ بالاعتبار

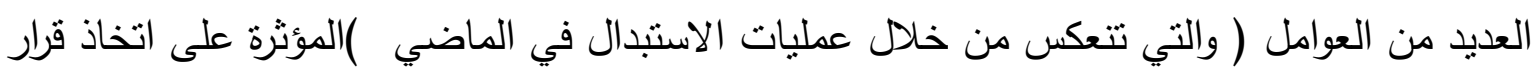
الاستبدال ويمكن تطبيق هذا النموذج على واحد او اكثر من الموجودات الانتاجية.

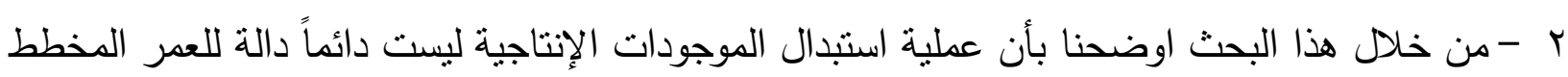

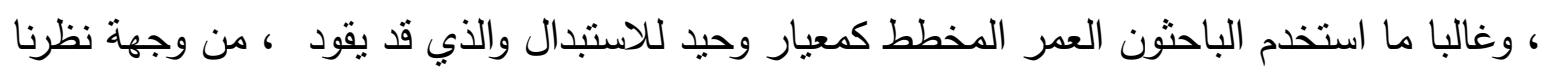

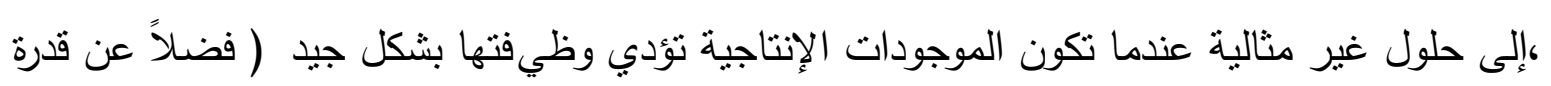
المعدات على الأداء بشكل جيد حتى بعد انتهاء العمر المخطط).

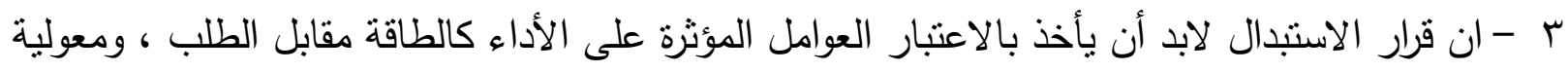

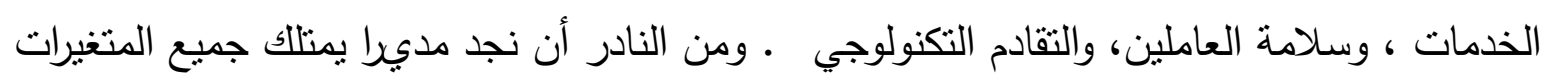

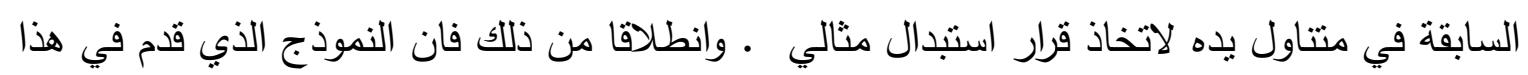

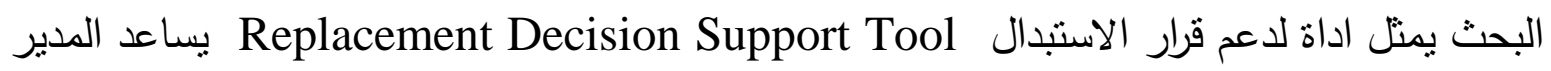


في إعداد جداول للاستبدال وتهيئة المستلزمات المالية لذلك. ولا ندعي بان هذا النموذج سيكون بديلا

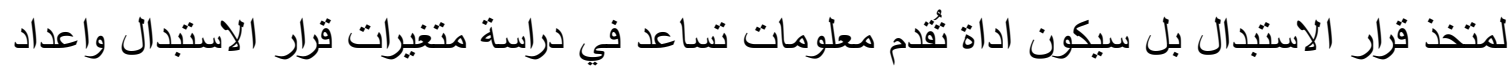

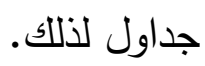

؛ - - إن سريان نتائج المحاكاة تعتمد على مصداقية التوزيع التجريبي لعطلية الاستبدال التي حدثت في

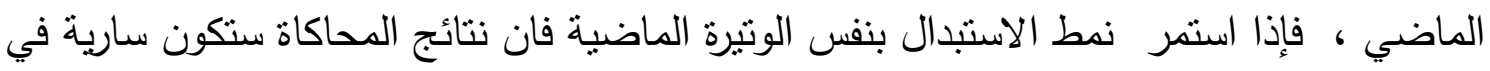

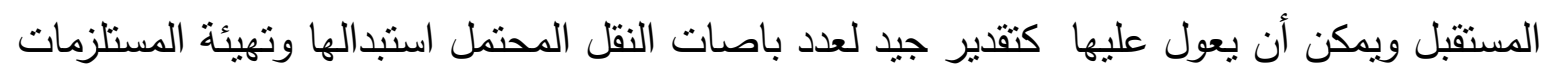

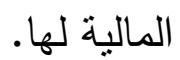

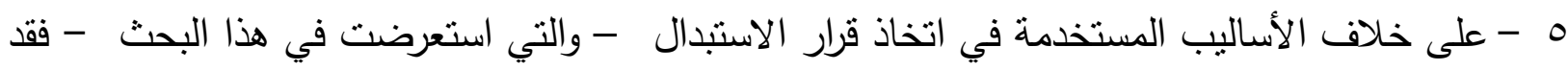

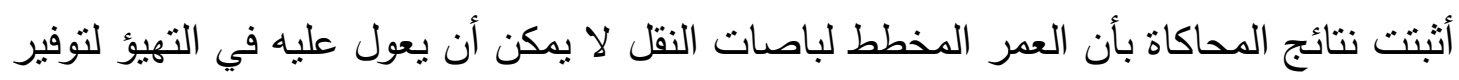

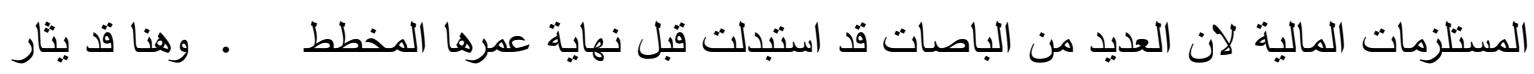

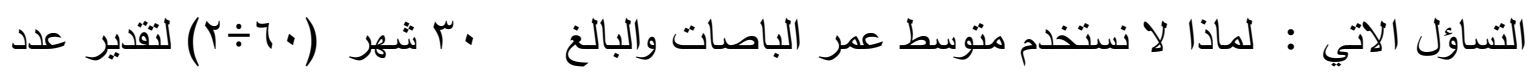

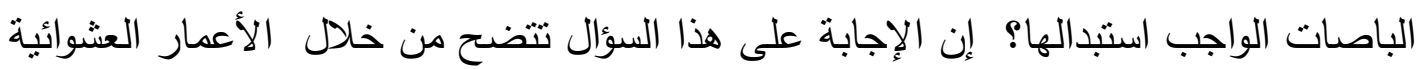

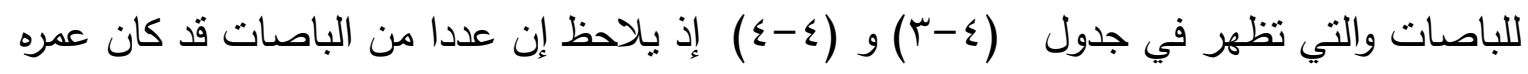

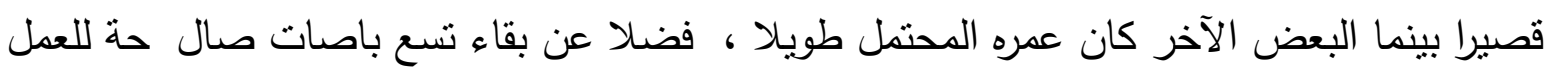
بعد انتهاء العمر المخطط لها.

7 - لا يقدم أي من اساليب التحليل المالية والكمية التي قدمت في هذا البحث فكرة عن العمر المحتمل

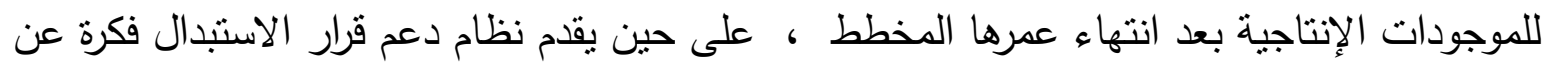
العمر المحتمل لتلك الموجودات بعد انتهاء العمر المخطط وذلك بنعكس على التنفقات النقدية المحتملة من الموجودات والطاقة الإنتاجية ايضاً.

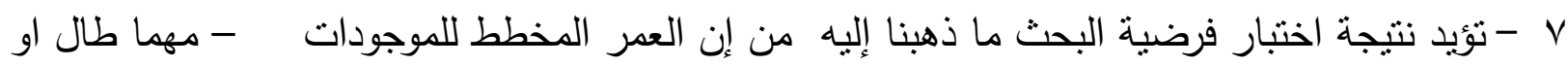
قصر - لا يؤثر على عمليات الاستبدال كون عملية الاستبدال تخضع لمتغيرات عديدة من غير العمر العنه المخطط. 
المصادر العربية

أ) الكتب

ا ل الربيعي، فاضل محسن وصلاح حمزة عبد، مقدمة في العمليات التصادفية ، مديرية دار الكتب للطباعة

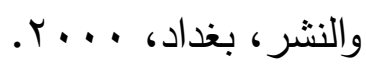

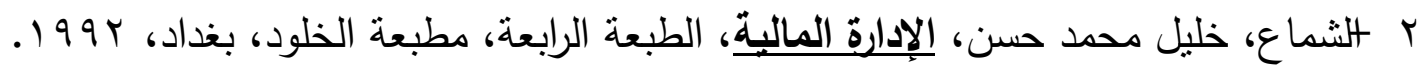

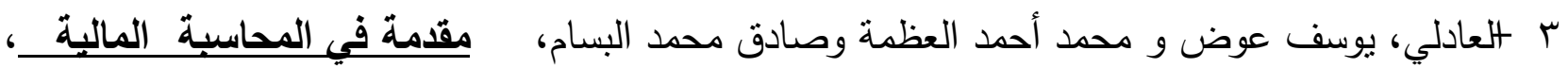
الطبعة الأولى، منشورات ذات السلاسل، 919 أو 19

ع رمضان، زياد، مبادئ الاستثمار الحقيقي والمالي_، الطبعة الأولى، دار وائل للطباعة والنشر، عمان، .1991 ه عبد الرحيم، علي عبد ويوسف العادلي ومحمد العظمة، أساسبات التكاليف والمحاسبة الإدارية ، الطبعة الأولى، منشورات ذات السلاسل، ـ9 19.

ب) الرسائل الجامعية

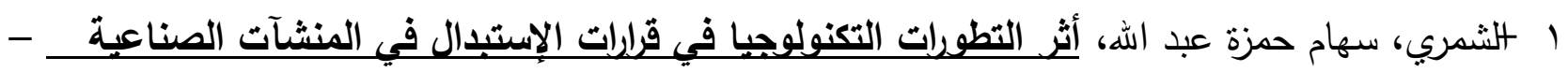
دراسة تطبيقية في المنشأة العامة للصناعات الصوفية، رسالة ماجستير ، كلية الإدارة والاقتصاد، جامعة بغداد، س99 19 ץ كسن، خالد حريجة، الصبانة والاستبدال للمكائن الإنتاجية - دراسة تطبيقية في المنشأة العامة لصناعة البطاريات السائلة، رسالة ماجستير، كلية الإدارة والاقتصاد، الجامعة المستتصرية، ـ99 19. ب - علم الدين، عماد حسام الدين، مقارنة تجرييية بين نماذج الإضافة الثابتة والإضافة المتغيرة للزمن لبعض أنظمة صفوف الانتظار، رسالة ماجستير، كلية الإدارة والاقتصاد، جامعة بغداد، 999 ا.

ا لالحبيطي، قاسم محسن، العلاقة بين الاندثار ودورة اكتساب الأرباح في عمليات اس تبدال الموجودات

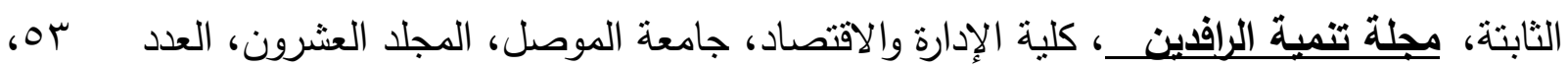
. (rVV-rY) (ص) 6 1991 r لثهيتي، خالد عبد الرحيم، وحكمت رشبد سلطان، استخدام النماذج الكمية في استبدال المعدات الإنتاجية،

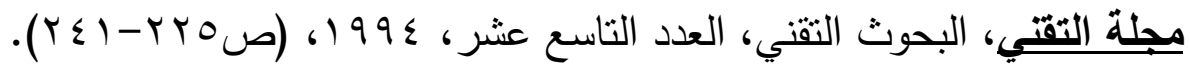
ب عبد المالك، عادل، وحسين سالم كيطان، تطبيقات الأساليب العلمية لاستبدال المكائن الإنتاجية، مجلة

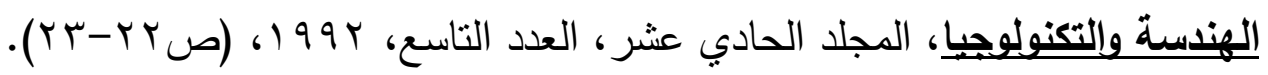

المصادر الأجنبية 
1- Ahuja, K.K., Production Management, $1^{\text {st }}$ ed., Prentice - CBC Publisher and Distributors, New Delhi, 1993.

2- $\quad$ Publishing, United States of America, 2001.

3- Bhttacharyya, S. K. and John Dearden, Accounting For Management: Text and Cases, $2^{\text {nd }}$ Ed., Vikas Publishing House Pvt. Ltd., New Delhi, 1996.

4- Bowman, Michael S., Applied Economic Analysis For Technologists, Engineers: and Managers, Prentice-Hall, Inc., United States of America, 1999.

5- Budnick, Frank S., Richard Mojena, and Thomas E. Vollmann, Principles Of Operations Research For Management, $1^{\text {st }}$ Ed., Richard D. Irwin, Inc., United States of America, 1977.

6- $\quad$ Evans, James R., Applied Production and Operations Management, $4^{\text {th }}$ ed., West Publishing Company, New York, 1993.

7- $\quad$ Heizer, Jay, and Barry Render, Principles Of Operations Management, $6^{\text {th }}$ Ed., Prentice-Hall, Inc., New Jersey, 2001.

8- $\quad$ Khan, M. Y., and P. K. Jain, Financial Management, $1^{\text {st }}$ Ed., Mc Graw-Hill Publishing Company, New Delhi, 1982.

9- Krajewski, Lee J., and Larry P. Ritzman, Operations Management: Strategy and Analysis, $3^{\text {rd }}$ Ed., Addision - Wesley Publishing Company, New York, 1993.

10- Krajewski, Lee J., and Larry P. Ritzman, Operations Management: Strategy and Analysis, $7^{\text {th }}$ Ed., Addision - Wesley Publishing Company, New York, 2005.

11- Lawrence, John A., and Barry Alan Pasternack, Applied Management Science: A computer-Integrated Approach For Decision-Making, John Wiley and Sons, Inc., New York, 1998.

12- Moore, Franklin G., and Thomas E. Hendrick, Production/ Operations Management, $8^{\text {th }}$ Ed., Richard D., Irwin, Inc., U.S.A., 1980.

13- Pandey, I. M., Management Accounting: A planning and Control Approach, $3^{\text {rd }}$ Ed., Vikas Publishing House Ptv Ltd., New Delhi, 1997.

14- Russell, Roberta S., and Bernard W. Taylor III, Production and Operations Management: Focusing On Quality and Competitiveness, Prentice-Hall, Inc., A Simon and Schuster Company, New Jersey, 1998.

15- Shafer, Scott M., and Jack R. Meredith, Operations Management: A process Approach with Spreadsheets, John Wiley and Sons, Inc., New York, 1998.

16- Shamblin, James E., and G. T. Stevens, Operations Research: a fundamental Approach, Mc Graw-Hill, Inc., New York, 1974.

17- Slack, Nigel, Stuart Chambers, Christine Harland, Alan Harrison and Robert Johnston, Operations Management, 4th Ed., Bitman Publishing Company, London, 2004. 
18- Sullivan, William G., James A. Bontadelli, Elin M. Wicks, and E. Paul De Garmo, Engineering Economic, $11^{\text {th }}$ Ed., Prentice-Hall, Inc., New Jersey, 2000.

19- Weston, J. Fred, Scott Besley, and Eugene F. Brigham, Essential of Managerial Finance, $11^{\text {th }}$ Ed., the Dryden Press Harcourt Barce College Publishers, New York, 1997.

1- D'aversa, J. S., and J. F. Shapiro, Optimal Machine Maintenance and Replacement by Liner Programming and Enumeration, Journal of the Operational Research Society, Vol. 29, No. 8, 1978, p. (759-768).

2- $\quad$ Gress, Edward J., Application of Replacement Cost Accounting: A case Study, A Journal of Accounting and Business Studies, Vol. 8, No. 1, 1972, p. (3-12).

3- Longo, M., and D. Zanobetti, Criteria for Asset Replacement Studies, Engineering Costs and Production Economics, No. 17, 1989, p. (271-275).

4- Nguyen, D. G., and D. N. P. Murthy, A combined Block and Repair Limit Replacement Policy, Journal of the Operational Research Society, Vol. 35, No. 7, 1984, p. (653-658).

5- $\quad$ Reid, Richard A., and Walter A. Stark, Optimal Replacement Policy Developed For Items That Fail, Industrial Engineering, Vol. 18, No. 3, 1986, p. (23-27).

6- Whitaker, David, An Investigation into the Decision Rules Of Capital Investment Using Simulation, Journal of the Operational Research Society, Vol. 35, No. 12, 1984, p. (1101-1111). 